Cognitive mechanisms underpinning statistical learning of grammatical regularities in child and adult learners: Evidence for a multicomponential view

Jelena Mirković1a, Marissa Yee ${ }^{2}$, Maddison Kennedy ${ }^{1}$, Marianna E. Hayiou-Thomas²

${ }^{1}$ School of Education, Language, and Psychology

York St John University

York, Y031 7EX

United Kingdom

2 Department of Psychology

University of York

York, Y010 5DD

United Kingdom

a Corresponding author:

jelena.mirkovic@york.ac.uk

All materials and data are available on osf.io/95z8y. 


\title{
Cognitive mechanisms underpinning statistical learning of grammatical regularities in child and adult learners: Evidence for a multicomponential view
}

\author{
Jelena Mirkovićia, Marissa Yee², Maddison Kennedy ${ }^{1}$, and Marianna E. Hayiou-Thomas ${ }^{2}$ \\ ${ }^{1}$ York St John University, ${ }^{2}$ University of York
}

\begin{abstract}
Statistical learning plays a key role in language acquisition and development, from word segmentation to grammar learning. In a recent review and meta-analysis, Frost et al. (2019) identified key contributions of the statistical learning literature over the last 20 years, as well as a number of limitations. Here we address three of those limitations across three experiments. First, we address the issue of unrealistic learning environments by training participants on an artificial language comprising multiple regularities (phonological, distributional, semantic), unlike the majority of previous statistical learning studies. Second, to examine learning at several levels of linguistic structure, we use a word learning paradigm at training, which allowed us to assess both word and grammar learning, including generalization of the trained regularities to previously unseen items. Third, to address the issue of underspecification of cognitive mechanisms underpinning statistical learning, we examine the emergence and role of explicit knowledge in generalization performance in child and adult learners. Additionally, we examine the role of off-line memory consolidation processes. Across three experiments and multiple tasks, we found that both children and adults showed good levels of word learning, but variable levels of generalization of the trained grammatical regularities. Generalization success depended on the age group, type of training, and type of regularity assessed. Across all three experiments, explicit knowledge of the regularities contributed to the performance in some generalization tasks, but it was not key for successful generalization. Off-line consolidation processes consistently influenced long-term maintenance of the newly acquired lexical knowledge, but evidence of their role in grammar learning was mixed. We argue that our findings shed light on the cognitive mechanisms underpinning statistical learning, and provide evidence in support of multicomponential views of statistical learning.
\end{abstract}

Keywords: statistical learning; implicit learning; explicit awareness; consolidation; artificial language; grammar 


\section{INTRODUCTION}

What is the knowledge that underpins our ability to distinguish between $a$ drink and to drink? One piece of information is the first word in the phrase: words following $a$ are likely to be nouns, and words following to are likely to be verbs (e.g., Redington et al., 1998). Grammatical categories such as nouns and verbs are the building blocks of linguistic knowledge. Significant evidence suggests that word co-occurrence cues to grammatical categories are learned via statistical learning, widely implicated across a range of phenomena as a key mechanism in language acquisition (e.g., Aslin \& Newport, 2012; Gómez \& Gerken, 2000; Monaghan et al., 2007; Reeder et al., 2013; see Lany \& Saffran, 2013 for review).

Word co-occurrence (distributional) information is just one type of statistical regularity that may be used to distinguish between different grammatical categories. In English, for example, nouns and verbs also differ in phonological properties such as stress position: English disyllabic nouns tend to have first syllable stress (a record, a permit), whereas English disyllabic verbs tend to have second syllable stress (to record, to permit; e.g., Kelly, 1992). Similar phonological cues to grammatical categories (e.g, phoneme type, phonological complexity, word length) have been identified cross-linguistically (e.g., Monaghan et al., 2007; Shi et al., 1998), and both child and adult learners use these cues in natural language acquisition and processing (e.g., Dikker et al., 2010; Farmer et al., 2006; Kelly, 1992; Monaghan et al., 2007). In studies using natural or artificial language learning paradigms it has been demonstrated that statistical learning also plays a role in learning these types of regularities (e.g., Cassidy \& Kelly, 1991, 2001; Gómez \& Lakusta, 2004; Monaghan et al., 2005).

In addition to distributional and phonological regularities, grammatical categories are associated with semantic regularities. For example, cross-linguistically nouns tend to refer to objects and entities, and verbs to actions and events (e.g., Gentner, 1982; Pinker, 1984). Nouns tend to be more concrete and imageable than verbs (e.g., McDonough et al., 2011). Semantic cues to grammatical categories are present even in such apparently arbitrary categories as grammatical gender, beyond the associations with natural gender (e.g., Mirković et al., 2005; Mirković et al., 2011; Zubin \& Köpcke, 1981, 1986). However, few statistical learning studies have examined the role of semantic cues in grammatical category learning; instead, they have focused on word learning (see Smith et al., 2014 for review). These studies have demonstrated that both infant and adult learners use statistical regularities in word-referent mappings across different contexts in learning word meanings (e.g., Smith \& Yu, 2008; Yu \& Smith, 2007, 2011). A small number of studies have examined the use of semantic regularities in grammatical category 
learning. For example, Lany and Saffran $(2010,2011)$ have shown that infants can use semantic regularities in grammatical category learning when these are correlated with additional phonological and distributional cues. Adult learners have similarly been shown to be able to learn grammatical gender-like categories partly based on semantic cues (e.g., Mirković et al., 2011; Mirković \& Gaskell, 2016).

Statistical learning is assumed to be an implicit learning mechanism that underpins the learning of these three types of linguistic regularities (e.g., Aslin, 2017; Aslin \& Newport, 2012; Christiansen, 2019; Kidd, 2012; Seidenberg \& MacDonald, 2018; Smith et al., 2014). Some evidence suggests that implicit learning emerges and matures early in development (e.g., Amso \& Davidow, 2012; Finn et al., 2016) and thus adults have often been used as proxies to examine the contribution of statistical learning mechanisms to language acquisition in infancy (e.g., Gómez, 2002; Newport \& Aslin, 2004; Reeder et al., 2013; Saffran et al., 1996, 1997; Vouloumanos, 2008; Yu \& Smith, 2007). However, more recent studies have indicated that, alongside implicit learning, explicit knowledge may emerge and support the learning in statistical learning paradigms (e.g., Batterink et al., 2015; Batterink et al, 2019; Franco et al., 2011; Monaghan et al., 2019; Smalle et al., 2018). For example, two studies have explored the role of implicit and explicit knowledge in statistical learning of phonological regularities. Using a standard statistical learning task with transitional probabilities as a cue for segmenting words in a speech stream, Batterink et al. (2015) showed that in adults both implicit and explicit knowledge influence the learning. Similar findings were reported by Smalle et al. (2018) in syllable sequence learning, using an adaptation of the Hebb repetition paradigm with child and adult learners. Interestingly, Smalle et al. (2018) found a developmental difference: the adults in their study were more strongly influenced by explicit knowledge than the children. Together, these studies suggest that both implicit and explicit mechanisms may play a role in the learning of form-based (phonological) statistical regularities used in speech segmentation and word learning.

In the current study our aim was to examine the contribution of explicit knowledge in statistical learning, focusing specifically on grammatical category learning. We used an artificial language encompassing multiple regularities and in this way mimicking grammatical systems in natural languages in that the regularities were simultaneously within phonological, distributional, and semantic properties of the new words. This is similar to several other studies exploring grammar learning and use (e.g., Amato \& MacDonald, 2010; Gómez \& Lakusta, 2004; Hopman \& MacDonald, 2018; Lany \& Saffran, 2010, 2011). Our study differs from the previous studies in that we examined both word and grammar learning, including generalization of the regularities 
to previously unseen items. As described in more detail below, we trained participants on the artificial language using a word learning task, and we assessed the learning of the grammatical regularities via a generalization task. Our rationale was that word and grammar learning proceed in parallel in natural language acquisition, and according to several theoretical frameworks grammar learning is dependent on lexical learning (e.g., Bates \& Goodman, 1997; Lany \& Saffran, 2013; Wonnacott, 2011). Hence our choice of the training paradigm and parallel assessment of word and grammar learning.

More broadly, our study aimed to address some of the limitations of the statistical learning literature identified in a recent review and meta-analysis by Frost and colleagues (2019). Across two decades of research and a number of key findings and theoretical contributions, Frost et al. (2019) identified several areas of weakness in the statistical learning literature. Here we focus on the following: First, a restricted range of methodologies and a narrow scope of research questions across the body of work were argued to risk limiting the broad theoretical relevance of the concept of statistical learning. Frost and colleagues suggested that this was partly due to the fact that real-world regularities were substantially more complex and multidimensional than the paradigms used in typical statistical learning studies (see also Siegelman et al., 2020). Second, Frost et al. argued that the theory of the cognitive mechanism(s) underpinning statistical learning was largely underspecified. The majority of studies subscribed to a unitary view of statistical learning as a single implicit learning mechanism, and frequently the theoretical construct of statistical learning was merged with the performance on the experimental task used to measure it. Frost and colleagues (Frost et al., 2015; Frost et al., 2019) provided some evidence that challenges the single-mechanism view, and argued for a multicomponential view suggesting that statistical learning comprises of both domain-specific mechanisms (e.g., within modality specific neural regions) and domain-general mechanisms that include both declarative/explicit and procedural/implicit memory systems (see Conway, 2020 , for a related proposal).

In the current study, we aimed to address three specific issues identified as limitations of the statistical learning literature by Frost and colleagues. First, our paradigm incorporated multiple regularities and thus provided a more realistic representation of the learning environment than the majority of previous statistical learning studies. Second, as natural language acquisition typically proceeds by simultaneous learning at several levels of linguistic representations, we examined both word and grammar learning within a single paradigm, again unlike the majority of statistical learning studies. We also assessed both the learning of the trained exemplars, and the ability to generalize the learned regularities to previously unseen items. Generalization is 
often taken as a hallmark of proficient linguistic behaviour, but in statistical learning studies item learning and generalization of regularities have often been studied separately (e.g., Gerken, 2010; Gerken et al., 2005; Gómez, 2002; Gómez \& Lakusta, 2004; Reeder et al., 2017; but see Frost \& Monaghan, 2016 for an exception). Third, we addressed the issue of underspecification of the cognitive mechanisms underpinning statistical learning by first, examining the contribution of explicit knowledge to what is typically assumed implicit learning; second, by assessing child and adult learners; and third, by examining the role of off-line memory consolidation processes.

Across three experiments, we trained adult and 10-year old child participants on an artificial language mimicking a grammatical gender system (Corbett, 1991; Mirković et al., 2011). Participants were trained using a word-learning task where each word (e.g., tib mofeem) consisted of a "determiner" (e.g., tib), a "stem" (e.g., mof), and a "suffix" (e.g., eem) and it was presented together with a picture of a familiar object (e.g., dog). Across the whole set of the trained words, there were three types of regularities. First, each word had one of the two "determiners" (tib or ked) and one of the two "suffixes" (eem or ool); these properties represented phonological regularities. Second, each tib word was paired with the suffix eem, and each ked word with the suffix ool (e.g., tib mofeem, tib hormeem; ked larshool, ked migool); this represented a distributional/co-occurrence regularity. Finally, all tib...eem words were presented with pictures of animals, and all ked...ool words with pictures of artefacts. This represented a regularity in the mapping between form and meaning. Across both adults and children, we systematically examined the learning of the words and of the statistical regularities, and the role of explicit knowledge in the generalization of the regularities to previously unseen items (the 'wugs' test; Berko, 1958). Across the three experiments, we varied the amount and type of training (Experiments 1-3), and we also assessed learning and generalization immediately after training, and after a short (24 hr) and a long (1 week) delay (Experiments 1 and 2) to examine consolidation and long-term retention.

\section{EXPERIMENT 1}

In Experiment 1, adults and children were trained on a set of new words implementing the grammatical system described above. We trained participants using a simple word learning task with no reference to the grammatical regularities (e.g., Dobel et al., 2010; Mirković \& Gaskell, 2016). We examined the learning of the regularities via generalization to previously unseen items. Our key aim was to systematically examine generalization patterns across different types 
of regularities, and the extent to which they were associated with levels of explicit knowledge of the regularities that may emerge in the course of learning.

Generalization was assessed by presenting participants with previously unseen novel words and pictures using the same word learning task as at training. Generalization items were either consistent with the trained regularities (i.e. new tib...eem words presented with pictures of new animals, new ked...ool words presented with pictures of new artefacts), or inconsistent with them. By manipulating the properties of the words and the pictures in the inconsistent trials, we assessed the learning of the mapping between the determiner and the suffix and the semantic category of the picture (Determiner + Suffix generalization test), and the learning of the suffix (Suffix Only generalization test) (the tests are described in detail below).

Explicit knowledge of the regularities was assessed using a verbal report at the end of the final testing session. Explicit knowledge of the determiner and of the suffix was scored separately using a scheme that assessed both the knowledge of the phonological form and the knowledge of the regularities in the form-meaning mapping.

In Experiment 1, we tested participants at three time points after learning to allow for any memory consolidation effects to emerge. Recent literature in word learning in children and adults has shown that neural processes that occur in off-line periods, and particularly during sleep, influence long-term representations of newly learned words (see James et al., 2017 for review). Some evidence suggests that children may show stronger consolidation effects in word learning than adults (e.g., Bishop et al., 2012; Weighall et al., 2017). The evidence of the influence of off-line consolidation in grammar learning is more mixed. Some studies have shown influence of sleep-related consolidation in the abstraction of distributional regularities (e.g., Gómez et al., 2006; Hupbach et al., 2009), and in implicit but not explicit measures of grammar learning (e.g., Tamminen et al., 2012). Studies combining word and grammar learning have shown consolidation effects in word but not grammar learning (e.g., Mirković \& Gaskell, 2016). However, unlike word learning studies, no grammar learning studies of which we are aware directly compared adult and child learners. Thus in the current study we explored the contribution of off-line consolidation in both word and grammar learning by testing adults and children at three time points: immediately after learning, and then after a short ( $24 \mathrm{hr}$ ) and a long (1 week) delay.

All participants were tested using the same protocol (Figure 1). At Session 1, they were taught the novel words using a word-picture matching task. Both word learning and the generalization 
of grammatical regularities were then assessed at three time points: immediately after learning at Session 1, and then after approximately 24 hours at Session 2 (short delay), and one week at Session 3 (long delay). All participants received training on the novel words only in Session 1. Explicit awareness of the regularities was assessed at the end of Session 3.

Method

All non-proprietary materials for all experiments are available on OSF (osf.io/95z8y).

\section{Participants}

A total of 43 participants took part in Experiment 1. Twenty three were typically developing, monolingual, native English-speaking children aged between 9 and 11 years (15 males, 12 females; mean age $=10: 09$, range $=9: 10-11: 07$ ) with no known language disorders, who were recruited from a local primary school in York, UK. They participated in the study with parental consent. Nineteen participants were monolingual, native English-speaking adults (8 males, 12 females; mean age $=21: 02$, range $=18: 07-28: 04$ ) with no known language disorders, who were recruited from the University of York community. They participated in the study after providing written informed consent.

The study was approved by the Ethics Committee at the Department of Psychology, University of York.

\section{Materials}

Training set: The training language consisted of 16 new words. Each new word consisted of a "stem" and a "suffix", both pronounceable English pseudowords selected from the ARC database (Rastle et al., 2002). There were 16 unique stems. Eight of the stems were combined with the suffix -eem (e.g., mofeem), and eight with the suffix -ool (e.g., larshool). Each of the -eem words was preceded by the "determiner" tib, and each of the -ool words by ked (e.g., tib hormeem, ked larshool).

The words were paired with pictures of familiar animals and objects (all pictures were normed for name agreement with a separate sample of children of the same age). The tib...eem words were paired with pictures of animals, and the ked...ool words with pictures of objects. There was no phonological overlap between the new word and the English word for the depicted entity. 
Generalization sets: Two additional sets of items were developed to test generalization of the regularities to new, previously unseen items.

In the Determiner + Suffix set, half of the new word-picture pairs were consistent with the regularities in the mapping between the determiner, the suffix, and the semantic category of the picture, and half were inconsistent. For example, for the consistent items a new tib...eem word was paired with a picture of a new animal, and a new ked...ool word was paired with a picture of a new object. Conversely, for the inconsistent items, a new tib...eem word was paired with a picture of a new object, and a new ked...ool word was paired with a picture of a new animal. Thus the inconsistent items matched the distributional regularities in the training set, but mismatched the form-meaning mapping.

In the Suffix Only set, half of the new word-picture pairs were consistent with all regularities in the training set, and half were inconsistent in the mapping between the suffix and both the determiner and the semantic category of the picture. Specifically, for inconsistent items a new eem word was paired with the determiner ked and a picture of an object, and a new -ool word was paired with the determiner tib and a picture of an animal. Thus the inconsistent items mismatched the distributional regularities (the co-occurrence between the determiner and the suffix) and the form-meaning mapping specifically in the suffix.

There was a total of 12 items in each generalization set, with 6 items in the consistent condition, and 6 in the inconsistent. Each set was divided into 3 subsets with 4 items each ( 2 consistent, 2 inconsistent), and a different subset was used for each of the three testing sessions.

All training and generalization words were presented in the spoken form only. They were digitally recorded in a sound-proof booth by a female native speaker of English.

\section{Procedure}

As illustrated in Figure 1, all participants followed the same protocol, with the training and an immediate test at Session 1, and two additional test sessions (with no additional training between the sessions). Session 2 was run 24-48 hrs after Session 1 (with all adults and 20 children tested after approximately 24 hours, and 3 children approximately 48 hours after Session 1). Session 3 was run approximately one week (7-8 days) after Session 1. All language tasks were presented using a custom-made iPad app. The order of the two generalization tests 
was counterbalanced across participants. All participants were additionally tested on three standardized tests (nonword repetition and two subtests from WASI II (Wechsler, 2011)), but these are not reported further.

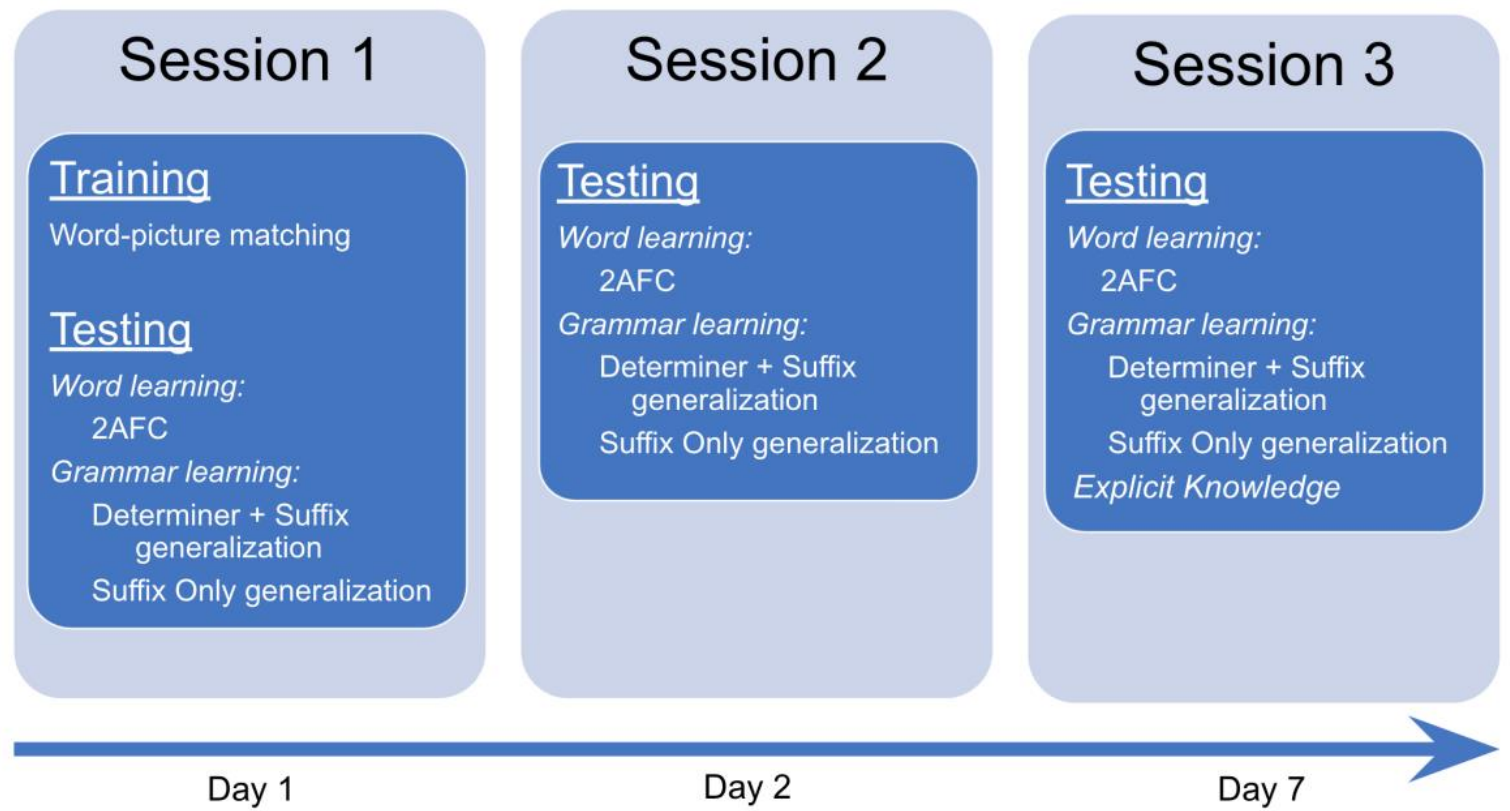

Figure 1. Experiment 1 design and tasks.

Training -- Word-picture matching: A word-picture matching task was used to train the new words and their meanings. Participants were presented with a cover story of an alien visiting Earth, and naming things he encountered using the Alien language. In each trial, a picture was presented in the center of the iPad screen, and a word was played over the iPad speakers. Two emoticons were presented in the corners of the screen below the picture, a smiley face in the right-hand corner, and a sad face in the left-hand corner. Participants were instructed to press the smiley face if they thought the picture and the new word 'go well together', and the sad face if they did not. The next word-picture pair was presented after the participant pressed one of the emoticons. The training on the regularities was implicit: no information was provided about any properties of the words or the pictures. Each word-picture pair was presented a total of 18 times, equally divided across 6 blocks of 3 repetitions per pair. The order of trials within the block was randomized for each participant. There were two practice trials before the main experimental trials. The training task took approximately 15-20 minutes to complete.

Tests - Word learning: A two-alternative forced choice (2AFC) task was used to test word learning. In each trial a target and a foil picture were presented in the center of the screen, and a trained word was played over the speakers. The foil pictures were other pictures from the 
training set, which were matched in the semantic category to the target picture. This ensured that the participant could not rely on the "grammatical" markers to establish the meaning of the word. The participant's task was to touch the picture on the screen that matched the word played over the speakers. Each experimental word was presented once, and each experimental picture was presented twice, once as the target, once as a foil. The experimental trials were preceded by two practice trials. This task took approximately 3-5 minutes to complete.

This task was administered 4 times, each time with different target-foil combinations. In Session 1, participants were tested on this task after 3 and again after 6 blocks of the word-picture matching training task. Each delayed test session started with the administration of this task. We used accuracy as the outcome measure.

Tests - Grammar learning: The same word-picture matching task used at training was used to test the ability to generalize the grammatical regularities to new items. The participant was reminded of the Alien cover story, and told that they would be presented with some more words from the Alien language. The new word-picture pairs from the two generalization sets were presented in separate blocks, with the order counterbalanced across participants. Within each block the order of trials was randomized for each participant. Each task took approximately 3 minutes to complete. We used a' as the outcome measure for this task (Pallier, 2002), with endorsements of word-picture pairs consistent with trained regularities considered hits, and endorsements of word-picture pairs inconsistent with the trained regularities considered false alarms. A' scores range from 0 to 1 , and account for response biases. A participant who endorsed both consistent and inconsistent items equally would have an a' score of .50; a participant who endorsed consistent pairs over inconsistent would have an a' score above .50, and a participant with the opposite pattern (inconsistent > consistent) an a' score below .50. We therefore took a' scores of above .50 to be evidence of learning of the regularities.

Explicit knowledge questionnaire: Participants' explicit knowledge was assessed in the final session (Session 3). Two questions were asked: one broad question asked whether they had any comments about the Alien language, and one specific question asked whether they used any strategies or clues to decide if the word and the picture matched. We opted for assessing levels of explicit knowledge as the very last task in the last session in order to avoid the testing itself influencing performance on the other tasks (e.g., Monaghan et al., 2019).

The responses on the explicit knowledge questions were scored separately for determiners and suffixes, to reflect the complexity of the knowledge of the regularities. A score of 3 was assigned 
to the responses which fully specified the regularities, including the form-meaning mapping. For the determiners, this would involve specifying that there were two determiners, and that tib was presented with the pictures of animals, and ked with the pictures of objects, and similarly for the suffixes. A score of 2 was assigned to the responses reflecting partial knowledge of the regularities. For the determiners, this would involve, for instance, fully specifying one of the determiners and with which category it was associated, and not fully specifying the second determiner, either in the form, or the form-meaning mapping. A score of 1 was assigned to the responses that identified that there were some regularities, but there was no reference to the form-meaning mapping. For example, for the determiners, this would involve specifying that some words started with tib and some with ked, but without any further specifications. A score of 0 was assigned when the response did not include any reference to the phonological or semantic regularities.

\section{Data analyses}

All data for all experiments are available on OSF (osf.io/95z8y).

All analyses reported below were performed in R (R Core Team, 2013).

In the analyses of word learning using the $2 \mathrm{AFC}$ task, we used one-tailed t-tests to compare average accuracy against chance (.5) in each session and each group. We also assessed group and test session effects using a mixed effects logistic regression (using glmer function in R) with accuracy coded as a binary outcome measure. Group was an effect coded fixed factor (adult $=.5$, child $=-.5$ ). Session was coded using Helmert contrasts, comparing performance after the long delay (Session 3) against performance at the immediate and the short delay (Session $1+$ Session 2), and then comparing the immediate session with the short delay (Session 1 vs. Session 2). Random effects were included for both participants and items, with a maximal inclusion of random slopes that allowed the model to converge (Barr et al., 2013). The mixed effects analysis was run using the lme4 package in $\mathrm{R}$ (Bates et al., 2015).

In the analyses of generalization performance, we used one-tailed t-tests to compare a' scores against 0.5 to assess the learning of the regularities. We also assessed group and session effects coded as above in a mixed effects linear regression (using lmer function in R) with a' as the outcome measure. 
In the analyses of explicit knowledge, we used levels of explicit knowledge of the affixes coded as described above as the outcome measure, group coded as above as a fixed factor, and affix as the second effect coded fixed factor (determiner $=.5$, suffix $=-.5$ ). We used multiple regression to assess the extent to which explicit awareness predicts performance on the generalization tests. For this analysis, explicit knowledge was group mean centered.

Results

Word learning

The performance on the 2AFC task was used to assess how well participants had learnt the meanings of the new words, and whether the level of learning differed between children and adults. We also tested the role of off-line consolidation by assessing changes in performance over time.

Figure 2 illustrates the level of learning for the trained items immediately after training (Session 1), and approximately 24 hours (Session 2) and one week later (Session 3) for both groups. Onesample t-tests confirmed that performance was significantly above chance for both children and adults in all three sessions (children; Session 1: $t(22)=6.07, p<.001$; Session 2: $t(22)=7.40, p$ $<.001$; Session 3: $t(22)=6.58, p<.001$; adults, Session 1: $t(18)=43.72, p<.001$; Session 2: $t$ $(18)=19.50, p<.001$; Session 3: $t(18)=21.35, p<.001$; Session 1: $\mathrm{M}_{\text {adults }}=.98, \mathrm{M}_{\text {children }}=.75$; Session 2: $\mathrm{M}_{\text {adults }}=.88, \mathrm{M}_{\text {children }}=.73$; Session 3: $\mathrm{M}_{\text {adults }}=.86, \mathrm{M}_{\text {children }}=.70$ ).
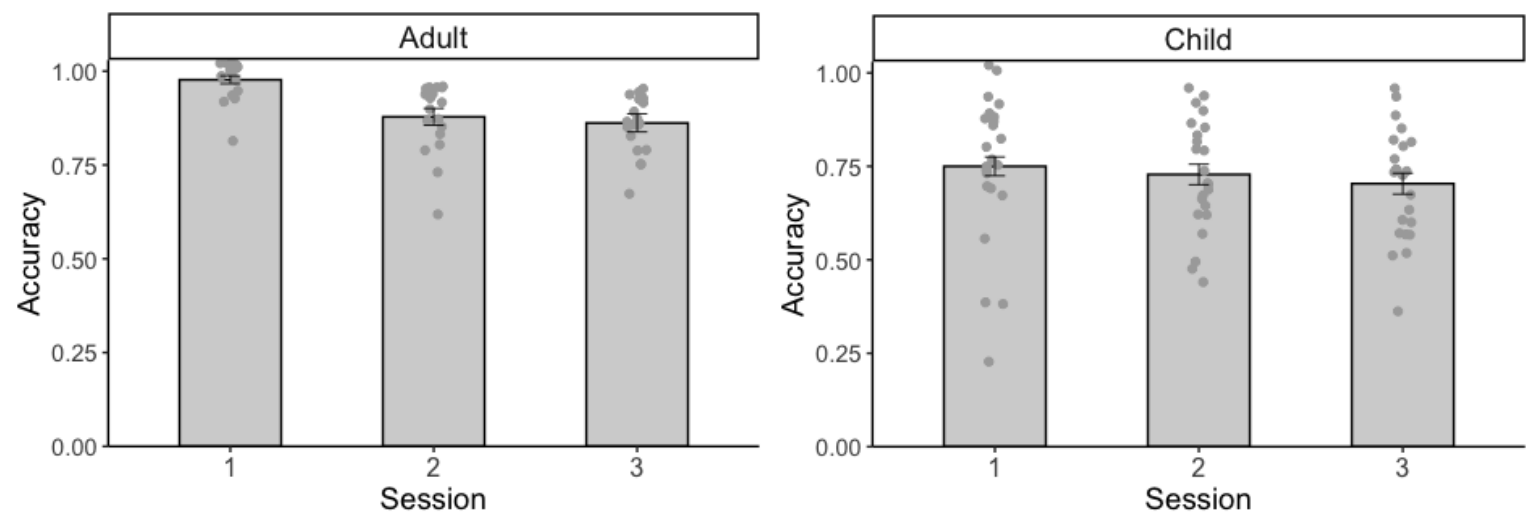

Figure 2. Accuracy (proportion correct) on the 2AFC task measuring word learning for adults and children immediately after learning (Session 1), approximately 24 hours (Session 2), and one week later (Session 3). 
As shown in Table 1 and illustrated in Figure 2, the results of the mixed effects regression analysis showed that adults had overall better word learning than children. In both groups there was evidence of forgetting over one week (session 3 vs. session $1+2$ ), but in both groups the memory for the novel words remained at or above $70 \%$ after a week-long delay and with no additional training. Interestingly, across both short and long delays children showed less forgetting than adults, as shown by the group x session 3 vs. session $1+2$, and group $\mathrm{x}$ session 1 vs. session 2 interactions.

Taken together, these results show that both adults and children showed good levels of learning of the new words that were maintained over the three sessions. While overall children learned fewer new items than adults, their memory for the new words was mostly maintained over the course of a week, whereas adults showed stronger forgetting over the same period.

Table 1. Regression coefficients for the fixed effects with accuracy as a binary outcome variable in the 2AFC word learning task.

\begin{tabular}{lllll} 
& Estimate & Std. Error & $\mathrm{z}$ & $\mathrm{p}$ \\
\hline Group & 1.95 & .35 & 5.61 & $<.001$ \\
Session 3 vs. $1+2$ & .37 & .13 & 2.87 & .004 \\
Session 1 vs. 2 & .43 & .24 & 1.81 & .071 \\
Group x Session 3 vs. 1+2 & .55 & .24 & 2.28 & .023 \\
& & & & \\
Group x Session 1 vs. 2 & 1.35 & .48 & 2.84 & .005 \\
& & & & \\
\hline
\end{tabular}

Random effects included in the model: intercepts by items and participants, and slope for Session 3 vs. $1+2$ by participants

Use of grammatical regularities: Generalization tests

We used generalization tests to assess the extent to which participants learned the grammatical regularities and were able to use them with previously unseen items. We assessed separately 
the performance on the determiner and suffix regularities (Determiner + Suffix test), and suffixes on their own (Suffix Only test). We calculated a' scores to index the level of generalization, with a' scores above 0.5 taken as evidence of the ability to generalize the knowledge to the new items. As in the word learning analyses, generalization performance was assessed at three time points: immediately after learning, after approximately 24 hours, and after one week.

\section{Determiner + Suffix test}

The Determiner + Suffix generalization test assessed whether participants were able to generalize the mapping between the tib ...eem and ked ...ool affixes and the semantic category (animals and objects respectively). We first examined whether the a' scores in each group and each session were greater than 0.5 , indicating the preference for the items consistent with trained regularities. Second, we tested group differences on this measure, and also the change over time.

One-tailed t-tests showed that as a group adults showed evidence of generalization in Sessions 1 and 2, but not in Session 3 (Figure 3; Session 1: $t(18)=4.28, p<.001$; Session 2: $t(18)=4.42, p$ $<.001$; Session 3: $t(18)=.90, p=.19)$. Unlike adults, children did not show evidence of having extracted the grammatical regularities, with none of the a' scores significantly different from 0.5 (Figure 3; children, Session 1: $t(22)=-0.95, p=.82$; Session 2: $t(22)=-1.13, p=.87$; Session 3: $t(22)=-0.59, p=.72)$.
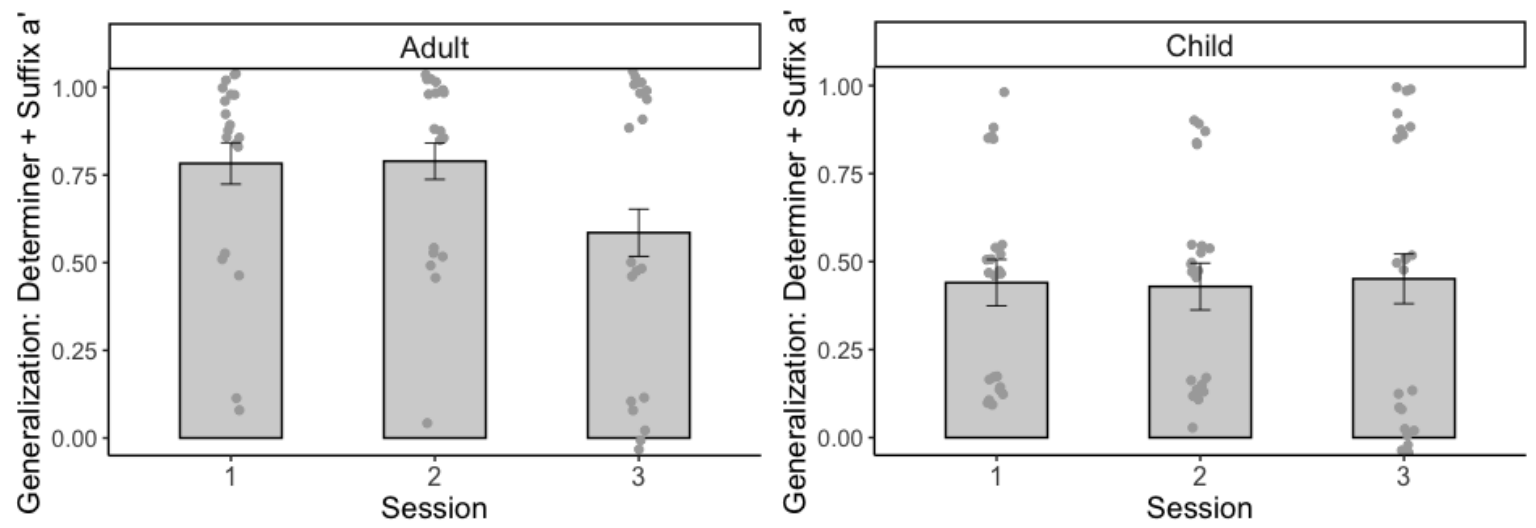

Figure 3. Performance on the Determiner + Suffix generalization test for the two groups across the three sessions.

The mixed effects regression assessing group and session effects confirmed that adults were overall significantly better in generalizing the trained regularities than children (group in Table 
2). There were no overall differences in performance across sessions, and there were also no reliable interactions between group and sessions (Table 2).

Table 2. Regression coefficients for the fixed effects with a' scores as the outcome in the Determiner + Suffix generalization test.

\begin{tabular}{lllll} 
& Estimate & Std. Error & $\mathrm{t}$ & $\mathrm{p}$ \\
\hline Group & & & & \\
& & & & \\
Session 3 vs. 1+2 & 0.06 & 0.04 & 1.53 & $>.1$ \\
Session 1 vs. 2 & 0.00 & 0.06 & 0.04 & $>.1$ \\
Group x Session 3 vs. 1+2 & 0.15 & 0.08 & 1.79 & .080 \\
& & & & \\
Group x Session 1 vs. 2 & -0.02 & 0.12 & -0.15 & $>.1$ \\
\hline
\end{tabular}

Random effects included in the model: intercept and slope for Session 3 vs. $1+2$ by participants

These findings confirm previous findings demonstrating that adults are able to learn and generalize a grammatical-gender like regularity in an artificial language (e.g., Mirković \& Gaskell, 2016). Despite evidence of some forgetting in individual word knowledge, adults' generalization performance was maintained over a 24-hour period. However, in parallel with a reduction in word knowledge after a longer delay, generalization performance was also reduced after a week long delay.

Unlike adults, children did not show evidence of successful generalization of this regularity, and their performance did not change after consolidation periods.

\section{$\underline{\text { Suffix Only test }}$}

The Suffix Only generalization test assessed whether participants had extracted the determinersuffix co-occurrence regularity, as well as the mapping between the suffix and the semantic category. 
Overall, the a' scores were lower in this task than in the Determiner + Suffix task (Figure 4). One-tailed t-tests indicated that neither adults nor children showed evidence of generalization in Sessions 1 and 2 (adults, Session 1: $t(18)=0.85, p=.20$; Session 2: $t(18)=1.21, p=.12$; children, Session 1: $t(22)=0.38, p=.35$; Session 2: $t(22)=0.46, p=.32)$. In Session 3 children's a' scores were reliably different from 0.5 , unlike those of adults (children, Session 3: $t(22)=$ 1.96, $p=.03$; adults, Session $3 t(18)=-.98, p=.83)$.

The mixed effects regression confirmed no overall differences between the groups in this task (group in Table 3), and revealed a non-reliable interaction between group and session 3 vs. 1+2, accounting for the increase in performance in children in the last session.
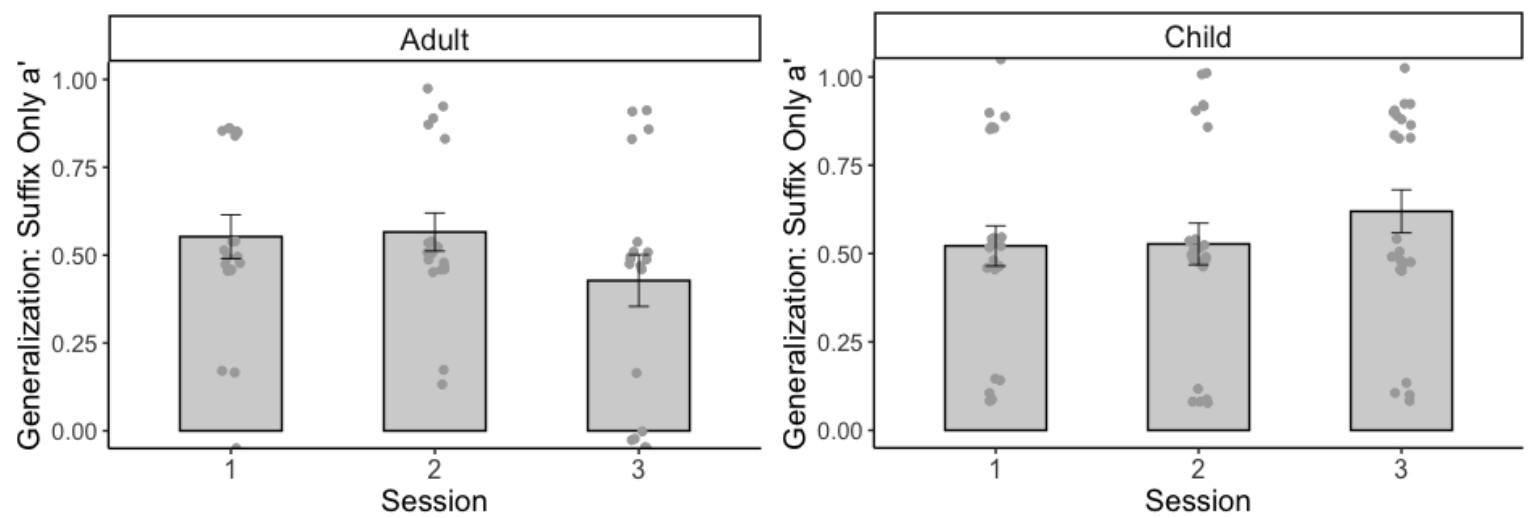

Figure 4. Performance on the Suffix only generalization test for the two groups across the three sessions.

Table 3. Regression coefficients for the fixed effects with a' scores as the outcome in the Suffix only generalization test.

\begin{tabular}{|c|c|c|c|c|}
\hline & Estimate & Std. Error & $\mathrm{t}$ & $\mathrm{p}$ \\
\hline Group & -0.041 & 0.051 & -0.800 & $>.1$ \\
\hline Session 3 vs. $1+2$ & 0.012 & 0.038 & 0.318 & $>.1$ \\
\hline Session 1 vs. 2 & -0.009 & 0.056 & -0.167 & $>.1$ \\
\hline Group x Session 3 vs. $1+2$ & 0.151 & 0.077 & 1.975 & 0.055 \\
\hline Group x Session 1 vs. 2 & -0.008 & 0.111 & -0.070 & $>.1$ \\
\hline
\end{tabular}


Random effects included in the model: intercept, and slope for Session 3 vs. $1+2$ by participants

These findings suggest that the generalization of the regularity involving the suffix only was more difficult than the regularity involving both affixes: neither adults nor children showed evidence of generalization in the first two test sessions. Adults' performance was stable across time, and there was some evidence of a consolidation-related change after a week-long delay in children.

Explicit knowledge of the regularities, and its relationship to performance on the generalization tasks

One of the main questions in the current study was about the extent to which participants would develop explicit knowledge of the grammatical regularities in the course of learning, and whether this knowledge would be associated with their performance in the generalization tasks. We found varying levels of generalization across the two tasks and the two groups, and in all cases there was significant individual variation (Figures 3 and 4). In the following analyses we examined first, overall levels of explicit knowledge of the regularities in children and adults, and second, the association between explicit knowledge and performance on the generalization tasks.

Levels of explicit knowledge of affixes in children and adults

Explicit knowledge was assessed in the final testing session (Session 3). Figure 5 illustrates the level of explicit knowledge of the determiner and the suffix in the two groups. A mixed effects analysis showed that in both groups there were greater levels of explicit knowledge of the determiners than the suffixes (affix: $\mathrm{b}=1.44, \mathrm{SE}=.17, \mathrm{t}(36)=8.76, \mathrm{p}<.0001$ ). Adults had greater levels of explicit knowledge than children (group: $b=1.07$, $\mathrm{SE}=.18, \mathrm{t}(36)=5.99, \mathrm{p}<$ .0001 ), and this was particularly pronounced with the determiners (group $\mathrm{x}$ affix: $\mathrm{b}=1.78, \mathrm{SE}=$ $.33, \mathrm{t}(36)=5.42, \mathrm{p}<.0001$; random effects included in the model: intercept and slope by participants).

These findings demonstrate that both children and adults show evidence of emergent explicit knowledge of some of the regularities implemented in the novel language. In both groups there were greater levels of explicit knowledge of the determiners than the suffixes, even though they 
occurred equally often during training. Adults showed significantly greater levels of explicit knowledge than children. Notably, adults' explicit knowledge of the determiners was particularly high $(\mathrm{M}=2.6$, out of a maximum possible score of 3$)$, in contrast to their performance on the generalization tasks assessed in the same session (Session 3). While as a group children showed significantly lower levels of affix knowledge than adults $\left(\mathrm{M}_{\text {determiner }}=\right.$ $0.65, \mathrm{M}_{\text {suffix }}=0.10$ ), there was individual variation in both age groups, as illustrated in Figure 5 .
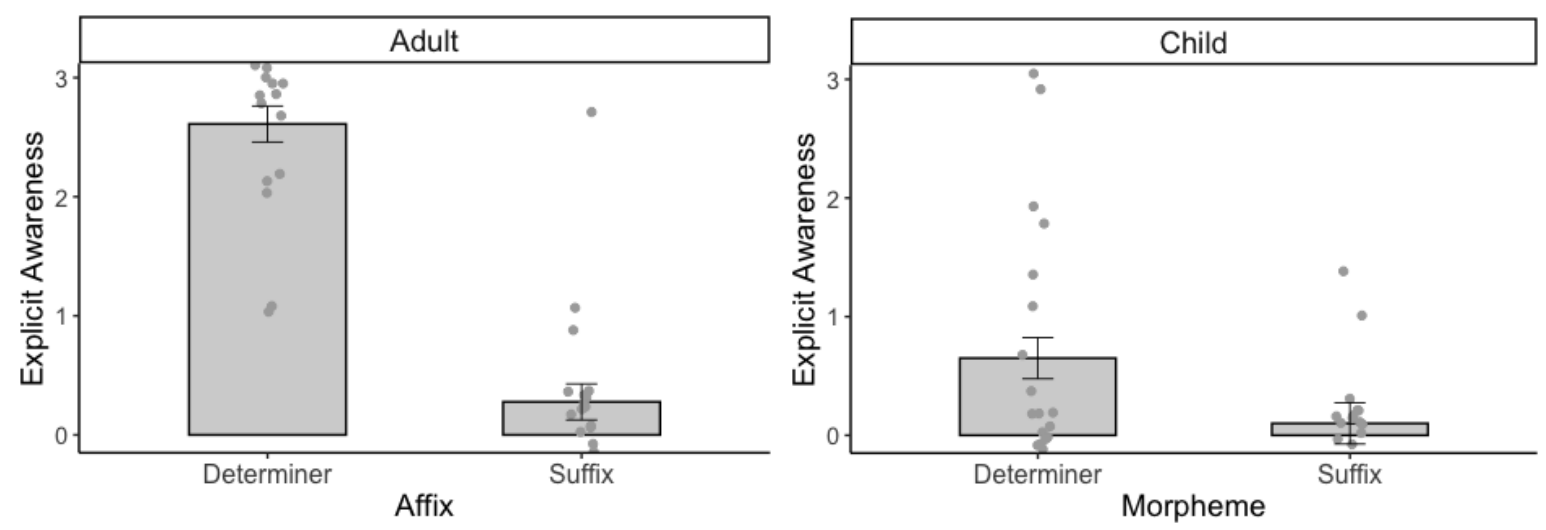

Figure 5. Levels of explicit awareness of determiners and suffixes in adults and children.

Association between explicit knowledge of the regularities and performance on generalization tasks

We next examined the extent to which explicit knowledge of the affixes was associated with generalization performance in both groups. We ran linear regression analyses to test the association between levels of explicit knowledge and performance on the Determiner + Suffix and Suffix Only generalization tasks for each test session. As the levels of explicit knowledge differed substantially between children and adults, we included both group and explicit knowledge as predictors. For each generalization test we included explicit knowledge of the affix relevant for the test: the determiner and the suffix for the Determiner + Suffix generalization test, and the suffix for the Suffix Only generalization test.

The first set of regression models used a' scores for the Determiner + Suffix tests in different sessions as the outcome variables, and group, explicit knowledge of the determiners, and explicit knowledge of the suffixes as predictor variables. For generalization performance in Session 1, the overall model accounted for $45 \%$ of the variance on the Determiner + Suffix test $\left(\mathrm{r}^{2}=.45\right.$, adjusted $\left.\mathrm{r}^{2}=.40, \mathrm{~F}(3,34)=9.29, \mathrm{p}<.001\right)$, and both group and levels of explicit knowledge of the affixes were reliably associated with the outcome (Table 4). Interestingly, 
explicit knowledge of the determiners had a facilitatory effect, whereas the explicit knowledge of the suffixes had an inhibitory effect. For generalization performance in Session 2, the overall model accounted for a significant $32 \%$ of the variance $\left(r^{2}=.32\right.$, adjusted $r^{2}=.26, F(3,34)=5.34$, $\mathrm{p}=.004$ ). As in Session 1, group was associated with generalization performance, but explicit knowledge of the affixes was not reliably associated with the outcome (Table 4). Finally, for generalization performance in Session 3 the overall model did not significantly predict variation in the performance on the Determiner + Suffix test $\left(\mathrm{r}^{2}=.12\right.$, adjusted $\mathrm{r}^{2}=.04, \mathrm{~F}(3,34)=1.57, \mathrm{p}=$ .215), and none of the predictors were reliably associated with the outcome (Table 4). These findings suggest that while explicit knowledge of the regularities emerges over the course of learning, and more so in adults than in children, good levels of explicit knowledge do not always result in good generalization performance. This is evidenced by good levels of explicit knowledge particularly of the determiners at Session 3 (Figure 5), but no evidence of successful generalization at the group level in the Determiner + Suffix task in the same session.

Table 4. Regression coefficients for Determiner + Suffix generalization as the outcome variable, and group and explicit knowledge of affixes as predictors.

\begin{tabular}{lcccc} 
Determiner + Suffix generalization & Estimate & Std. Error & $\mathrm{t}$ & $\mathrm{p}$ \\
\hline & & & & \\
Session 1 & & & & \\
Group & 0.34 & 0.09 & 3.99 & $<.001$ \\
Determiner knowledge & 0.13 & 0.05 & 2.64 & .012 \\
& & & & \\
Suffix knowledge & -0.19 & 0.08 & -2.45 & .019
\end{tabular}

Session 2

$\begin{array}{lcccc}\text { Group } & 0.32 & 0.09 & 3.43 & .002 \\ \text { Determiner knowledge } & 0.05 & 0.05 & 0.91 & .370 \\ & & & & \\ \text { Suffix knowledge } & -0.17 & 0.09 & -1.92 & .063\end{array}$




$\begin{array}{lcccc}\text { Group } & .075 & 0.13 & 0.58 & .563 \\ \text { Determiner knowledge } & 0.13 & 0.07 & 1.78 & .084 \\ & & & & \\ \text { Suffix knowledge } & 0.11 & 0.12 & 0.93 & .360\end{array}$

The second set of regression models examined generalization performance on the Suffix Only test in different sessions as the outcome variables, with group and explicit knowledge of the suffix as predictors. Unlike the Determiner + Suffix test, generalization performance on the Suffix only test was not reliably associated with any of the predictors at any of the test sessions (Session 1: total $\mathrm{r}^{2}=.04$ (adjusted $\mathrm{r}^{2}=.02$ ), $\mathrm{F}(2,35)=.66, \mathrm{p}=.521$; Session 2: total $\mathrm{r}^{2}=.06$ (adjusted $\left.\mathrm{r}^{2}=.01\right), \mathrm{F}(2,35)=1.18, \mathrm{p}=.319$; Session 3: total $\mathrm{r}^{2}=.11$ (adjusted $\left.\mathrm{r}^{2}=.05\right), \mathrm{F}(2,35)=$ $2.06, \mathrm{p}=.142$; see Table 5 for the contributions of individual predictors).

Table 5. Regression coefficients for Suffix Only generalization as the outcome variable, and group and explicit knowledge of the suffixes as predictors.

Suffix Only generalization $\quad$ b $\quad$ Std. Error $\quad t \quad p$

Session 1

$\begin{array}{lcccc}\text { Group } & 0.07 & 0.09 & 0.79 & .433 \\ \text { Suffix knowledge } & -0.07 & 0.08 & -0.84 & .408\end{array}$

Session 2

$\begin{array}{lllll}\text { Group } & 0.06 & 0.09 & 0.66 & .515\end{array}$

$\begin{array}{lllll}\text { Suffix knowledge } & 0.11 & 0.08 & 1.39 & .173\end{array}$

Session 3

$\begin{array}{lllll}\text { Group } & -0.20 & 0.10 & -1.95 & .060\end{array}$ 


\section{Discussion}

In Experiment 1 we explored statistical learning of grammatical regularities by training participants on a novel language incorporating multiple regularities. The regularities mimicked a grammatical gender-like system found in many natural languages. Our aims were to examine generalization patterns across different regularities in child and adult learners, and to shed more light on the cognitive mechanisms underpinning statistical learning. Specifically, we examined whether explicit knowledge of the regularities emerged in the course of learning and off-line consolidation, and the extent to which it was associated with generalization performance. We also examined whether off-line consolidation influenced word and grammar learning in both groups.

Overall, the results of Experiment 1 indicate that while both adults and children were able to learn the novel words to a good level, levels of generalization of the regularities incorporated in the training language were more variable. First, adults as a group were able to generalize the Determiner + Suffix regularity in the first two sessions but not the last one, and showed no evidence of generalization of the Suffix Only regularity in any of the sessions. This confirms previous findings using a similar paradigm, showing greater difficulty with generalization of the less salient affix (Mirković \& Gaskell, 2016; see also Culbertson et al., 2017; Frigo \& McDonald, 1998). Second, despite good levels of novel word learning and unlike adults, children as a group showed little evidence of generalization of the regularities, with only weak evidence of the ability to generalize in the Suffix Only test at the last session. This is a surprising finding, given previous evidence of the ability of younger children to learn and generalize similar types of regularities (e.g., Lany \& Saffran, 2010, 2011). We return to this issue in Experiments 2 and 3.

By the end of the final session, participants in both groups developed some level of explicit knowledge of the grammatical regularities. Adults' explicit knowledge was greater than the children's, which may partly be due to the nature of our test requiring an explicit verbal report. Both children and adults showed greater explicit knowledge of the determiners than the suffixes, and adults in particular showed high levels of explicit knowledge of the determiners. When examining the relationship between levels of explicit knowledge of the affixes and generalization, there were two notable findings. First, explicit knowledge was not consistently 
associated with generalization performance: we found some evidence of the association in the Determiner + Suffix test, but not in the Suffix Only test. This could be due to low levels of explicit knowledge of the suffixes in both groups and no strong evidence of generalization in the Suffix Only test (although we note significant individual variation in both). Second, explicit knowledge of the determiners was not associated with the generalization performance in a simple way. In adults, despite high levels of explicit knowledge of the determiners as assessed at Session 3, we did not find evidence of successful generalization in the Determiner + Suffix test in the same session at the group level. However, level of explicit knowledge of the determiners assessed at Session 3 was a significant predictor of generalization performance at the Determiner + Suffix test in Session 1. This pattern of associations may suggest that participants who were good at generalizing immediately after training might also be more likely to develop explicit knowledge after a week-long consolidation period. Together, these findings suggest that while generalization performance and explicit knowledge of the regularities are related, explicit knowledge does not seem to be the key driver of the performance in the generalization tasks.

We also examined the effects of off-line consolidation. We found stronger effects in word than grammar learning in that in both groups there was evidence of forgetting of the novel words over the delay, that was stronger in adults than in children. This developmental difference is similar to the pattern found in Smalle et al. (2018) over both a week and a year long delays. Importantly, in our study both groups maintained good levels of word knowledge over the week-long period, with no additional training. In grammar learning there was weak evidence of the influence of consolidation in children (weak evidence of generalization after a week long delay in the Suffix Only test). In adults, if generalization was successful immediately after training as in the Determiner + Suffix test, it was maintained over a short delay but reduced over a week-long delay. If generalization was unsuccessful as in the Suffix Only test there was no evidence of improvement over either a short or a long delay. The lack of improvement during off-line consolidation in grammar learning converges with previous findings using a similar paradigm (e.g., Mirković \& Gaskell, 2016).

As children did not show strong evidence of successful generalization of the regularities in Experiment 1, in Experiments 2 and 3 we further examined the factors that may influence grammar learning and generalization in a paradigm encompassing multiple regularities.

\section{EXPERIMENT 2}


One surprising finding from Experiment 1 was that child learners showed little evidence of the ability to generalize the statistical regularities. Previous studies in children showed that patterns of generalization may vary in artificial languages with complex or probabilistic regularities (e.g., Boyd \& Goldberg, 2012; Wonnacott, 2011; Wonnacott et al., 2012; Wonnacott et al., 2017). However, the regularities in the language we used were fully consistent and predictable: all tib words ended with the eem 'suffix', and all ked words with ool; all tib...eem words were paired with pictures of animals, and all ked...ool words with pictures of objects. Further, the language implemented similar regularities as studies with younger children who showed evidence of generalization (e.g., Lany \& Saffran, 2010, 2011).

One factor known to influence the learning of grammatical regularities is variability of exemplars. For example, in the classic study by Gomez (2002) exploring the learning of distributional regularities, adults and 18-month old infants were trained on a language with a co-dependency between the first and the third element in a sequence (aXb), similar to the codependency between the determiner and the suffix in the language used in Experiment 1 above. Gomez manipulated the number of second elements (X in aXb) in the training set, and showed that in both adults and infants the learning of the non-adjacent dependency (a...b) improved as the number of middle elements at training increased. Gomez argued that while a greater number of middle elements increased variability in the local dependencies, it actually increased the invariance of the non-adjacent dependencies, making them easier to learn.

To assess the extent to which a greater number of exemplars may enhance children's generalization in our paradigm, in Experiment 2 we increased the number of stems in the training set. We examined whether this change would improve children's performance, and additionally whether an increased number of stems may influence adult performance particularly with the generalization of the less salient suffix cue.

As there was no strong evidence of consolidation processes after the long delay enhancing generalization of the grammatical regularities, in Experiment 2 we only tested participants immediately after learning and after a 24 hour delay. Explicit knowledge was again assessed in the final (delayed) session. This allowed us to assess the extent to which explicit knowledge of the regularities emerges after a short 24 hour delay.

Method 


\section{Participants}

A total of 53 participants took part in Experiment 2. Thirty were typically developing monolingual native English-speaking children aged between 9 and 10 years (18 females; mean age $=10 ; 00$, range $=9 ; 04-10 ; 06)$ with no known language disorders. The children were recruited from local primary schools in Yorkshire, UK, and participated in the study with parental consent. Twenty two participants were monolingual, native English-speaking adults (23 females; mean age $=20 ; 08$, range $=18 ; 07-25 ; 03$ ) with no known language disorders. The adult participants were recruited from the York St John University community. They participated in the study after providing written informed consent. The study protocol was approved by the School of Psychological and Social Sciences Ethics Committee at York St John University.

\section{Materials}

Training set: We increased the size of the training language from 16 (Experiment 1) to 24 new words. The structure of the words and the language was the same as in Experiment 1, with the tib...eem words presented with pictures of familiar animals, and the ked...ool words with pictures of familiar objects. The only difference from Experiment 1 was in the number of stems, with 12 each in the tib...eem and ked...ool groups. The additional stems and pictures were a subset of the items used in the final generalization test in Experiment 1.

Generalization sets: We used the same two types of generalization sets as in Experiment 1 (Determiner + Suffix, and Suffix Only) to test participants' ability to generalize the grammatical regularities to new items. The structure of the two sets was the same as in Experiment 1. There was a total of 8 items in each set, divided into 2 subsets with 2 consistent and 2 inconsistent items in each. A different subset of items was administered at the two testing points (Session 1 and Session 2).

\section{Procedure}

As in Experiment 1, all participants followed the same protocol, with training and testing in Session 1, and testing only in Session 2 (Figure 6). Session 2 tasks were administered approximately 24 hours after Session 1 for all participants. 


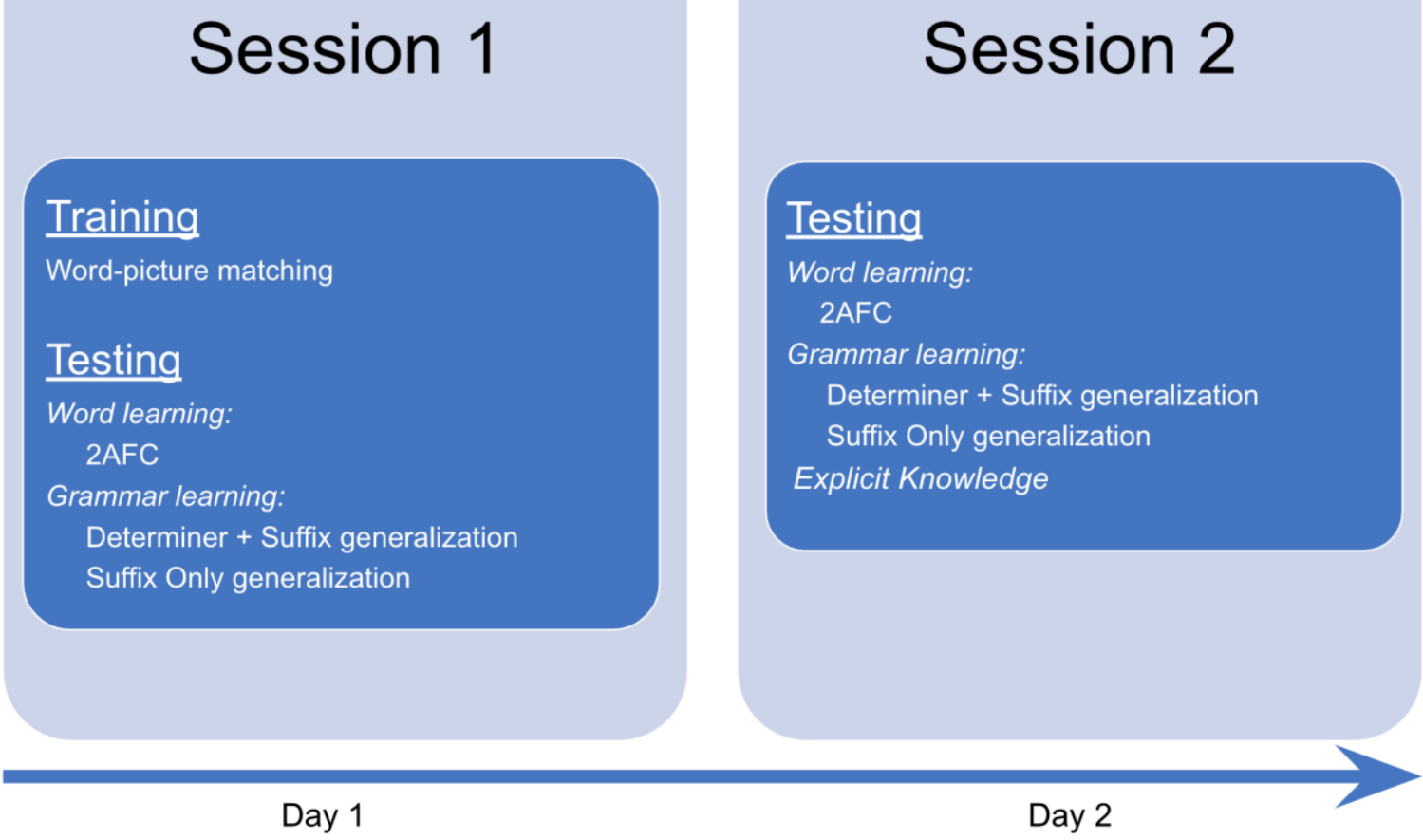

Figure 6. Experiment 2 design and tasks.

Training -- Word-picture matching: All experimental tasks were implemented in DMDX (Forster $\&$ Forster, 2003), and were administered on a laptop computer. The same cover story was used for the training task as in Experiment 1. Each trial started with a fixation cross presented at the center of the screen for $500 \mathrm{~ms}$ which was replaced by the target picture accompanied by the new word played over the laptop speakers. Participants were asked to press the keyboard key marked with a smiley sticker if they thought the picture and the word 'went well together', and the key marked with a sad emoticon sticker if they thought they did not. The laptop keyboard was used to record the responses, with the smiley sticker placed on the '?/' key, and the sticker on the ' $z$ ' key. As the number of trained words to be learned was greater in Experiment 2 than in Experiment 1, in order to maintain similar levels of word learning across the two experiments we increased the number of exposures of each word-picture pair from 18 in Experiment 1 to 27 in the current experiment (divided across 9 blocks of 3 repetitions each). The order of trials was randomized for each participant. There were two practice trials for this task. The training task took 35-40 minutes to complete.

Tests - Word learning: As in Experiment 1, we used a 2AFC task to test participants' learning of the word meanings. Each trial started with a presentation of the fixation cross at the center of the screen for $500 \mathrm{~ms}$, which was replaced by two pictures, one in each half of the screen, for $800 \mathrm{~ms}$, when one of the new words was played (the pictures remained on the screen). 
Participants were asked to press the 'yellow' button (the '+=' key was marked with a yellow sticker) if they thought the picture on the right matched the word, and the 'blue' button (the '1!' key was marked with a blue sticker) if they thought it was the picture on the left. As in Experiment 1, each new word was presented once. The foil pictures were from the same semantic category, and different target-foil pictures were used at different testing points. This task took approximately 5 minutes to complete. In Session 1, this task was administered twice, once after 5 blocks of word-picture matching, and once at the end of training. We used accuracy on this task as a measure of word learning.

Tests - Grammar learning: As in Experiment 1, we tested the ability to generalize the grammatical regularities to new items using the same task as for training, the word-picture matching task. We used a' scores as the outcome variable.

Explicit Knowledge questionnaire: We used the same two questions as in Experiment 1, administered in Session 2, to assess participants' explicit knowledge of the grammatical regularities.

\section{Data analyses}

We ran the same data analyses as in Experiment 1. All fixed factors were effect coded (group: child $=-.5$, adult $=.5$; session: Session $1=-.5$, Session $2=.5$; morpheme: determiner $=.5$, suffix $=$ $-.5)$. The random effects are reported with each analysis below.

Results

Word learning

As in Experiment 1, word learning was assessed using the 2AFC task. Children had similar levels of word learning as in Experiment 1, and adults slightly reduced (Figure 7; Session 1: $M_{\text {adults }}=$ $.84, \mathrm{M}_{\text {children }}=.75$; Session $2: \mathrm{M}_{\text {adults }}=.86, \mathrm{M}_{\text {children }}=.75$ ). For both groups and both sessions the performance on the $2 \mathrm{AFC}$ task was significantly better than chance (adults, Session 1: $t(22)=$ 12.40, $p<.001$; Session 2: $t(22)=12.93, p<.001$; children, Session 1: $t(29)=8.59, p<.001$; Session 2: $t(29)=8.03 p<.001)$. As in Experiment 1 , adults showed overall better word learning than children (Table 6). In both groups, there was no evidence of a change in 
performance across the delay (no main effect of session, and no reliable group $\mathrm{x}$ session interaction (Table 6)), suggesting good maintenance of word knowledge.
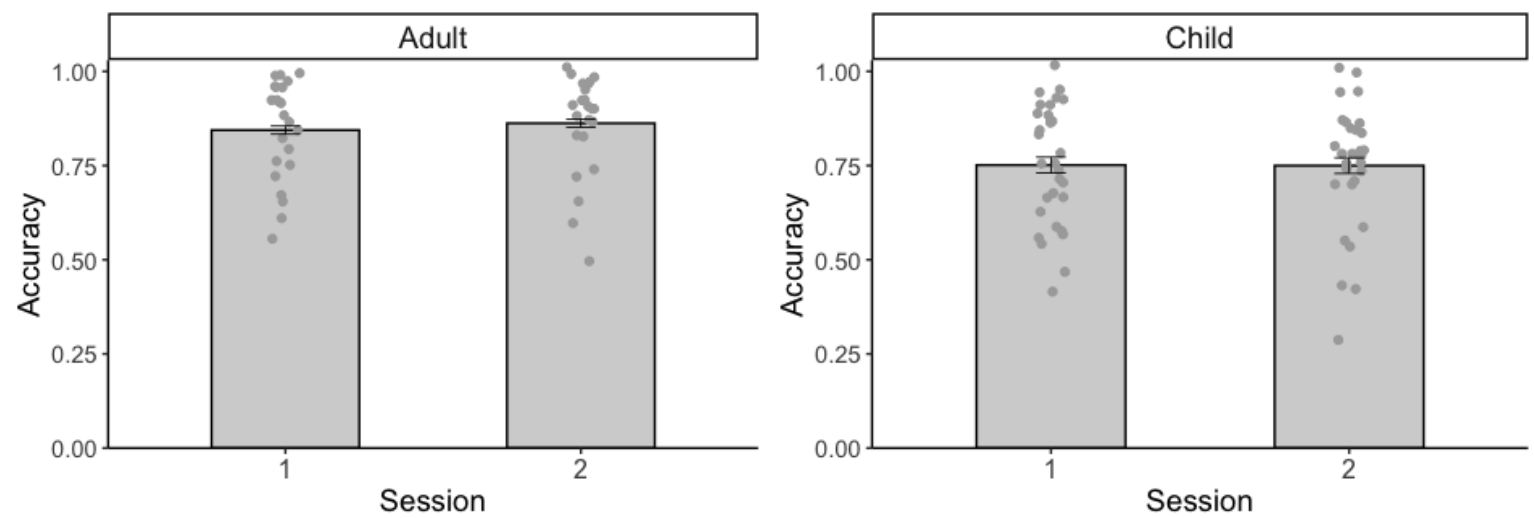

Figure 7. Accuracy on the 2AFC task measuring word learning for adults and children immediately after learning (Session 1) and approximately 24 hours later (Session 2).

Table 6. Regression coefficients for the fixed effects with accuracy as the binary outcome variable in the 2AFC word learning task.

\begin{tabular}{lllll} 
& Estimate & Std. Error & $\mathrm{z}$ & $\mathrm{p}$ \\
\hline Group & .85 & .27 & 3.09 & .002 \\
Session & .09 & .15 & .62 & .536 \\
Group x Session & .18 & .27 & .67 & .505 \\
\hline
\end{tabular}

Random effects included in the model: intercepts by items and participants, and slopes for session by participants and for group and session by items.

Use of grammatical regularities: Generalization tests

The key aim of Experiment 2 was to examine the extent to which an increased number of exemplars may influence generalization performance. Below we present the results from the two generalization tests.

Determiner + Suffix test 
As illustrated in Figure 8, children's a' scores in the Determiner + Suffix test were similar to those obtained in Experiment 1, whereas adults' were lower than those we had previously found. When compared against 0.5 , for adults there was evidence of generalization only in Session 2 but not Session 1 (Session 1: $t(22)=.52, p=.305$; Session 2: $\mathrm{t}(22)=2.24, \mathrm{p}=.018$ ). Children's performance was similar to that in Experiment 1 in that there was no evidence of generalization, despite an increased number of stems and the same level of word learning (Session 1: $t(29)=-.29 p=.61$; Session 2: $\mathrm{t}(29)=-.75, \mathrm{p}=.771)$. In a mixed-effects analysis with group and session as fixed effects, there were no reliable contributions from any of the predictors (Table 7).
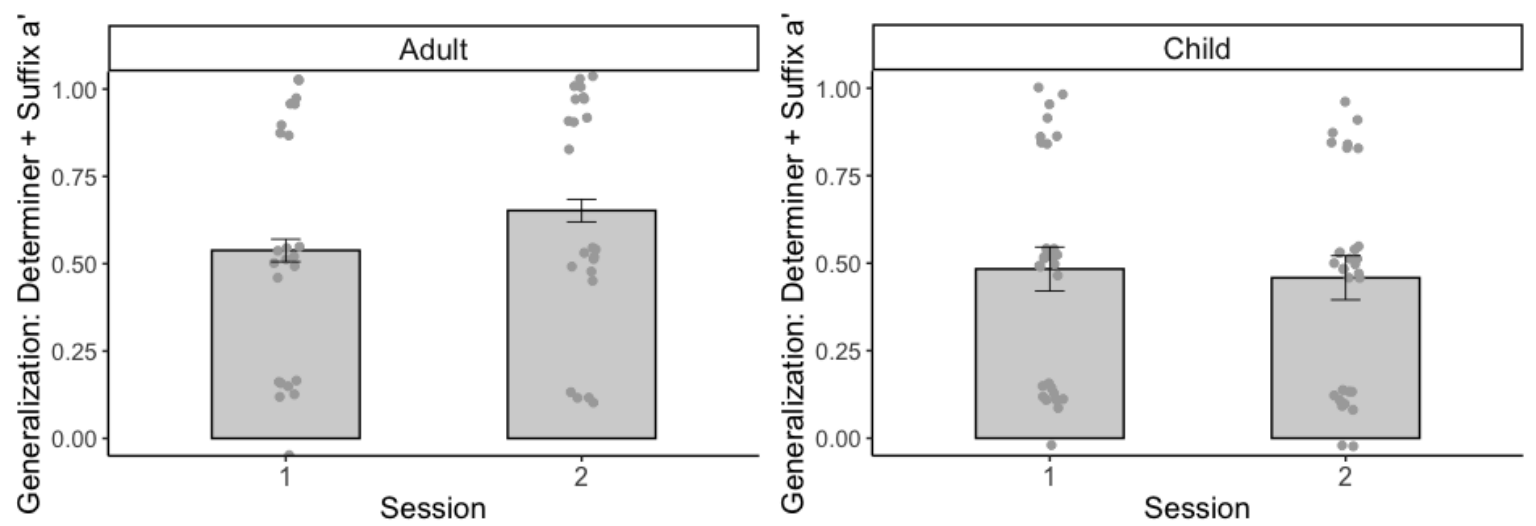

Figure 8. Performance on the Determiner + Suffix generalization test for the two groups across the two sessions.

Table 7. Regression coefficients for the fixed effects with a' scores as the outcome in the Determiner + Suffix generalization task.

\begin{tabular}{lllll} 
& Estimate & Std. Error & $\mathrm{t}$ & $\mathrm{p}$ \\
\hline Group & .12 & .07 & 1.75 & .087 \\
Session & -.04 & .05 & -.82 & .419 \\
Group x Session & -.14 & .11 & -1.27 & .209 \\
& & & & \\
\hline
\end{tabular}

Random effects included in the model: intercept by participants.

$\underline{\text { Suffix Only test }}$ 
There was no reliable evidence of generalization in the Suffix Only test in either group and in either session, as assessed by one-tailed t-tests against 0.5 (adults: Session 1: $t(22)=1.54, p=$ .069; Session 2: t (22) = 0, p = .5); children: Session 1: $t(29)=.21, p=.412$; Session 2: t (29) = $.202, \mathrm{p}=.579$; Figure 9). The mixed effects analysis showed no significant effects (Table 8).
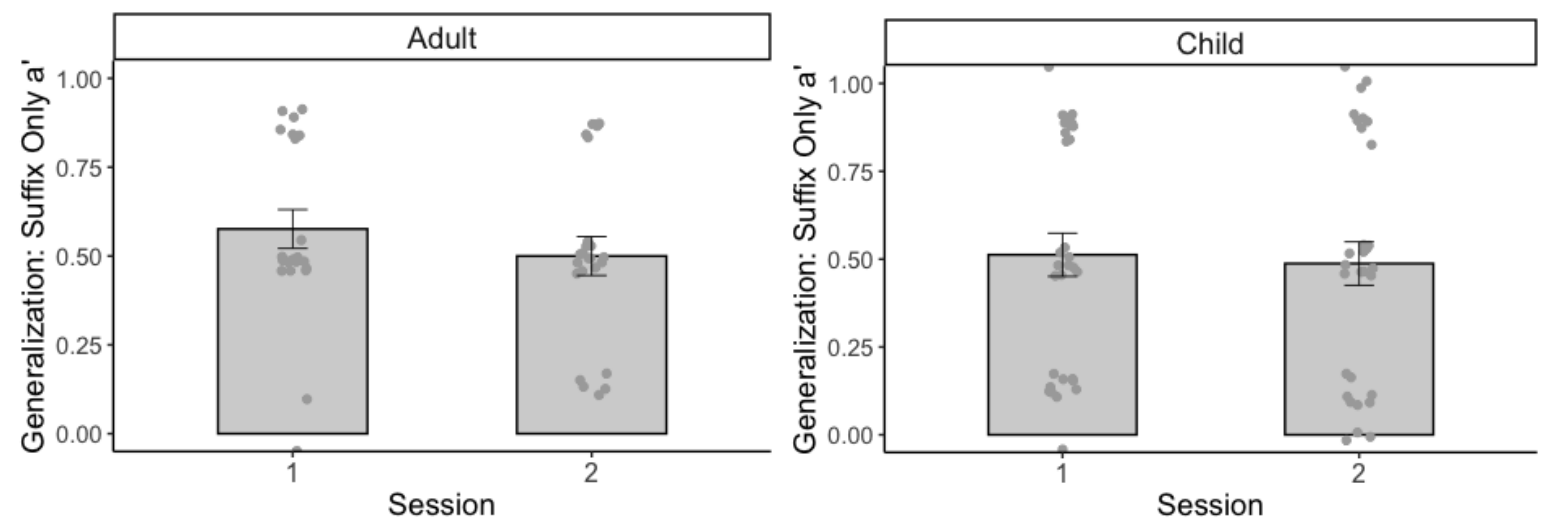

Figure 9. Performance on the Suffix only generalization test for the two groups across the two sessions.

Table 8. Regression coefficients for the fixed effects with a' scores as the outcome in the Suffix only generalization task.

\begin{tabular}{lllll} 
& Estimate & Std. Error & $\mathrm{t}$ & $\mathrm{p}$ \\
\hline Group & .04 & .06 & .65 & .518 \\
Session & .05 & .05 & .86 & .391 \\
Group x Session & .05 & .12 & .44 & .664 \\
\hline
\end{tabular}

Random effects included in the model: intercept by participants.

In sum, contrary to our predictions, an increase in the number of stems did not improve generalization performance in either group and either generalization test. Children's performance was similar to Experiment 1, whereas adults' performance was lower, and successful generalization was only observed in the Determiner + Suffix test administered 24 hours after learning. 
Explicit knowledge of affixation patterns, and its relationship to performance on the generalization tests

Levels of explicit knowledge of affixes in children and adults

As illustrated in Figure 10, and as in Experiment 1, adults showed greater levels of explicit knowledge than children $(\mathrm{b}=.55, \mathrm{SE}=.14, \mathrm{t}=3.89, \mathrm{p}<.001)$, and this was more pronounced for the determiners than the suffixes (group $\mathrm{x}$ affix interaction: $\mathrm{b}=1.08, \mathrm{SE}=.283, \mathrm{t}=3.82, \mathrm{p}<$ .001). Similar to Experiment 1, levels of explicit knowledge were greater for the determiners than for the suffixes, where there was little evidence of explicit knowledge in both groups (affix: $\mathrm{b}=.94, \mathrm{SE}=.14, \mathrm{t}=6.65, \mathrm{p}<.0001$ ). Overall, levels of explicit knowledge were lower in Experiment 2 than in Experiment 1 (adults: $\mathrm{M}_{\text {determiner }}=1.52, \mathrm{M}_{\text {suffix }}=0.04$; children: $\mathrm{M}_{\text {determiner }}=$ $\left.0.43, \mathrm{M}_{\text {suffix }}=0.03\right)$.
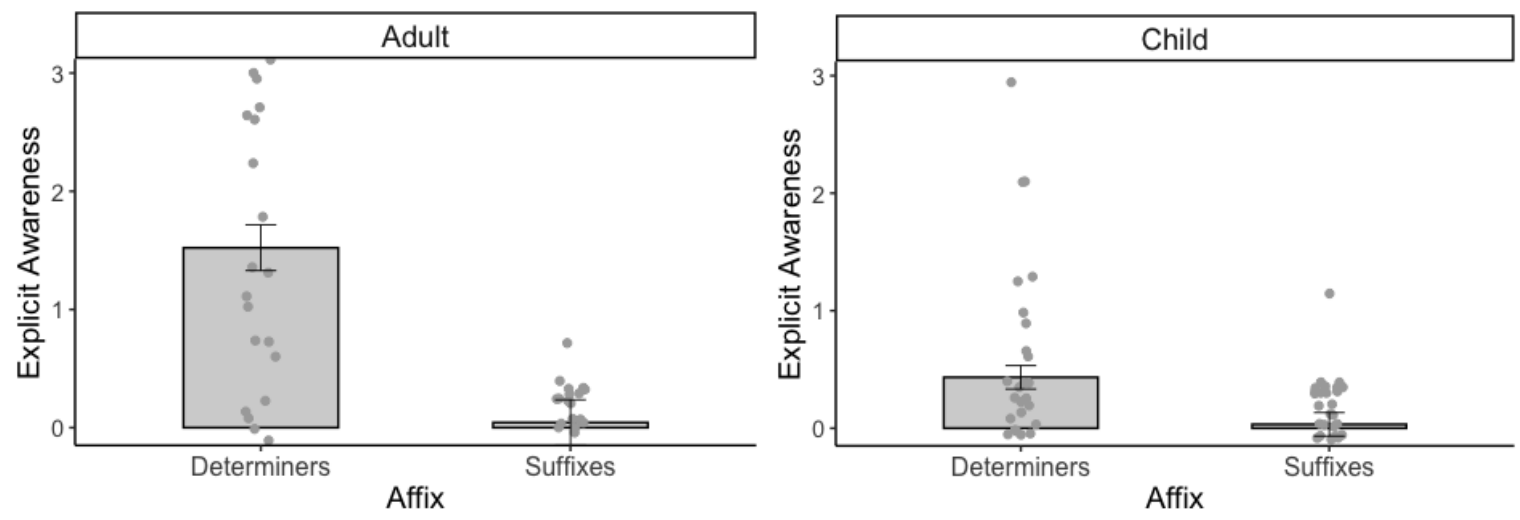

Figure 10. Levels of explicit knowledge of determiners and suffixes in adults and children.

Association between explicit knowledge of the regularities and performance on generalization tests

As in Experiment 1, we used linear regression analyses to assess the extent to which levels of explicit knowledge of the determiner and the suffix assessed at the final (delayed) test session (Session 2) predicted performance in the generalization tests at both the immediate (Session 1) and the delayed session. While we did see an overall decrease in generalization performance relative to Experiment 1, there was significant individual variation in performance in both tests and both sessions (Figures 8 and 9). 
With a' scores in the Determiner + Suffix generalization test at Session 1 as the outcome, the model including group, explicit knowledge of the determiner and explicit knowledge of the suffix accounted for a non-significant $2 \%$ of the variance (total $r^{2}=.02$, adjusted $r^{2}=.04, F(3,49)$ $=.37, p=.77$ ). None of the predictors were associated with the outcome (Table 9). However, with generalization performance in the same task in Session 2 as the outcome, the overall model accounted for a significant 19\% of the variance (total $\mathrm{r}^{2}=.19$, adjusted $\mathrm{r}^{2}=.14, \mathrm{~F}(3,49)=3.73, p$ $=.017)$. Group and explicit knowledge of the determiner were both significant predictors, but explicit knowledge of the suffixes was not (Table 9).

Table 9. Regression coefficients for Determiner + Suffix generalization as the outcome variable, and group and explicit knowledge of affixes as predictors.

Determiner + Suffix generalization $\quad$ Estimate $\quad$ Std. Error $\quad t \quad p$

$\begin{array}{lllll}\text { Session } 1 & & & & \\ \text { Group } & .05 & .09 & .58 & .562 \\ \text { Determiner knowledge } & .04 & .05 & .86 & .400 \\ & & & & \\ \text { Suffix knowledge } & .07 & .24 & .30 & .764\end{array}$

Session 2

Group

.19

.08

$2.32 \quad .025$

Determiner knowledge

.10

.04

$2.40 \quad .020$

Suffix knowledge

$-.01$

.22

$-.03 \quad .975$

None of the predictors were associated with the performance on the Suffix Only task in either session (Session 1: total $\mathrm{r}^{2}=.01$, adjusted $\mathrm{r}^{2}=.03, \mathrm{~F}(2,50)=.32, p=.73$; Session 2: total $\mathrm{r}^{2}=.00$, adjusted $\mathrm{r}^{2}=.00, \mathrm{~F}(2,50)=.01, p=.99$; see Table 10 for individual predictors $)$. 
Table 10. Regression coefficients for Suffix Only generalization as the outcome variable, and group and explicit knowledge of the suffixes as predictors.

\begin{tabular}{lllll} 
Suffix Only generalization & Estimate & Std. Error & $t$ & $p$ \\
& & & \\
\hline
\end{tabular}

Session 1

$\begin{array}{llllll}\text { Group } & .06 & .08 & .77 & .44\end{array}$

$\begin{array}{lllll}\text { Suffix knowledge } & -.05 & .21 & -.22 & .83\end{array}$

Session 2

$\begin{array}{lllll}\text { Group } & .01 & .09 & .15 & .88\end{array}$

$\begin{array}{lllll}\text { Suffix knowledge } & .01 & .22 & .03 & .98\end{array}$

Discussion

Based on evidence that increased variability might enhance the learning of grammatical regularities, in Experiment 2 we increased the number of exemplars in the training language. In order to help maintain the levels of word learning similar to Experiment 1, we also increased the number of repetitions of individual words at training. This training regime resulted in similar levels of word learning in children, but a somewhat reduced level of word learning in adults. Additionally, this training regime did not improve generalization in children, and it led to a decrease in generalization in adults. Importantly, despite no improvement in generalization in children, and a reduction in adults, we again found evidence of emerging explicit knowledge of the regularity involving the determiner, which contributed to successful generalization in adults in Session 2.

There are several possible reasons why an increased number of exemplars did not improve generalization performance. First, as in the original study by Gomez (2002) where improvements were found with a set size of 24 but not 12 (relative to 2 and 6), it is possible that an even greater number of exemplars was needed to improve generalization in our paradigm which contained phonological and semantic regularities in addition to the distributional 
regularities. Second, the increased number of repetitions of individual word-picture pairs might have enhanced the focus on the form-meaning mappings relevant for learning the specific word meanings (tokens) at the expense of the type-based, grammatical regularities (e.g., Vlach et al., 2012; Werchan \& Gómez, 2014). This is particularly evident when comparing adult performance in Session 2 in Experiments 1 and 2. (Performance in the word learning task in Session 1 is less directly comparable between the two experiments because of the difference in the total duration of the training blocks.) In this session, the level of word knowledge in the two experiments is comparable (86\% correct in Experiment 2, 88\% correct in Experiment 1), but the generalization performance in the Determiner + Suffix task in Experiment 2 is notably lower relative to Experiment 1 (a' of 0.65 vs. 0.79). This finding can be interpreted within the proposals for the role of forgetting of irrelevant features in generalization (e.g., Mirković et al., 2019; Vlach et al., 2012; Werchan \& Gómez, 2014). For example, Werchan and Gomez (2014) trained 2.5-year old children in a word learning task, where children learned labels for novel categories of objects. Crucially, the novel objects were presented in different contextual backgrounds and the children's ability to generalize the labels to new exemplars was tested after a delay that included sleep or wake. Werchan and Gomez argued that forgetting of irrelevant features (in this case, contextual backgrounds) may be crucial for generalization, and as sleep is more likely to enhance individual, contextualized, item memories (e.g., Mirković et al., 2019), they argued that children in the wake condition may be better able to generalize the labels. This is exactly what they found. Thus, in the paradigm we used in Experiment 2 with an increased repetition of the individual word-picture pairs, this enhanced exposure to individual items might have emphasized the unique features of the form-meaning mappings relevant for word learning at the expense of the regularities relevant for generalization.

To address the possible issue that increased repetition of individual word-picture pairs contributed to the decrease in generalization performance, in Experiment 3 we reduced the exposure to individual word-picture pairs, but took advantage of the production effect -- the enhancement of learning by introducing a language production element to the training (e.g., Hopman \& MacDonald, 2018; MacLeod \& Bodner, 2017; Zamuner et al., 2016).

\section{EXPERIMENT 3}

The "production effect" -- enhanced memory for words that have been pronounced, written, or typed -- is a well established phenomenon in memory research (see MacLeod \& Bodner, 2017 for review). A number of factors are thought to contribute to this effect including additional 
exposure from both producing and hearing one's own speech, increased demands of language production, and retrieval practice (e.g., Hopman \& MacDonald, 2018; Karpicke \& Roediger, 2008). Hopman and MacDonald (2018) recently examined the production effect in the context of language learning, specifically focusing on the role of language production mechanisms. They hypothesized that due to the involvement of utterance planning processes (including temporary maintenance, serial ordering, and binding across different levels of linguistic representations) the use of language production at training might be particularly beneficial for learning semantic or phonological co-dependencies between words. In their study participants learned an artificial language comprising novel nouns, verbs, articles, and adjectives, within sentences with a specific word order, and phonological and semantic co-dependencies between the novel words. Participants were trained using either a comprehension or a production-focused paradigm, while controlling for other factors (e.g., frequency of exposure). Production-focused training resulted in significantly better learning of both the vocabulary and the grammar of the novel language. Importantly, the production-based training had beneficial effects for word order and interword co-dependency learning even after the new vocabulary knowledge was taken into account.

Building on the findings from the study by Hopman and MacDonald (2018), in Experiment 3 we used the same extended training set as in Experiment 2 but instead of the enhanced exposure to individual word-picture pairs we included a language production component in the training task. Based on Hopman and MacDonald's proposal, we hypothesized that using language production at training may improve the learning of the grammatical regularities in our paradigm. We used the same generalization tests as in Experiments 1 and 2, and included an additional test to assess generalization of the determiner-suffix distributional co-occurrence regularity, without reference to the semantic cues. We included two additional tests of word learning, one focusing on the phonological form (old/new judgements), and one involving a language production component (picture naming). The latter test allowed us to assess the learning of both the vocabulary and the grammatical regularities in the trained items. In Experiment 3 we tested participants only immediately after training.

Method

Participants 
A total of 61 participants took part in the study. Twenty eight were monolingual children, native speakers of English, aged between 9 and 11 years (mean age $=10.03$ ), with no known language disorders. They were recruited from primary schools in Yorkshire, UK, and participated in the study with parental consent. Thirty three were adult monolingual, native speakers of English, with no known language disorders, recruited from the York St John University community (mean age $=28.03$ ). They participated in the study after providing written informed consent. The study protocol was approved by the York St John University School of Psychological and Social Sciences Ethics Committee.

\section{Materials}

Training set: The same training set was used as in Experiment 2. We additionally developed 24 items as foils for the new Phonological form old/new task used to assess word learning (described below). These items followed the same design principles as the training set.

Generalization sets: We used three generalization sets in Experiment 3. For the Determiner + Suffix and the Suffix Only sets we combined the items from the two sessions in Experiment 2, resulting in 8 items ( 4 consistent, 4 inconsistent) in each set.

A new set of items was developed for the Phonological Form Only generalization test. This test aimed to assess the distributional regularity (the determiner and suffix co-occurrence (tib... eem; ked... ool)), with no reference to the semantic regularity. It consisted of an additional set of 8 new word forms (and no accompanying pictures). Four of these were consistent with the trained distributional regularities, and four were inconsistent (e.g., tib...ool, ked...eem).

The words from all three generalization sets were digitally recorded by a native speaker of English.

\section{Procedure}

The overall procedure was similar to Experiments 1 and 2, with two key differences. First, all participants were trained and tested in the same session and there were no delayed tests. Second, we introduced four additional tasks. One was introduced at training (Word Repetition), two were additional tests of word learning (Phonological Form Old/New judgement task, 
Picture Naming), and one included the additional set of generalization items (Phonological Form Only generalization) (Figure 11).

The training phase consisted of the Word Repetition and Word-Picture Matching tasks. Word learning was assessed using the 2AFC task at the end of training, and using the Phonological Form Old/New judgment task and Picture Naming administered after the generalization tasks. The three generalization sets were used to assess generalization performance. The order of the three sets was counterbalanced across participants. All training and test tasks were implemented in DMDX. Explicit knowledge of the affixes was assessed at the end of the session using the same questions as in Experiments 1 and 2. The whole session lasted approximately 1 hour, and participants were encouraged to take breaks between the tasks.
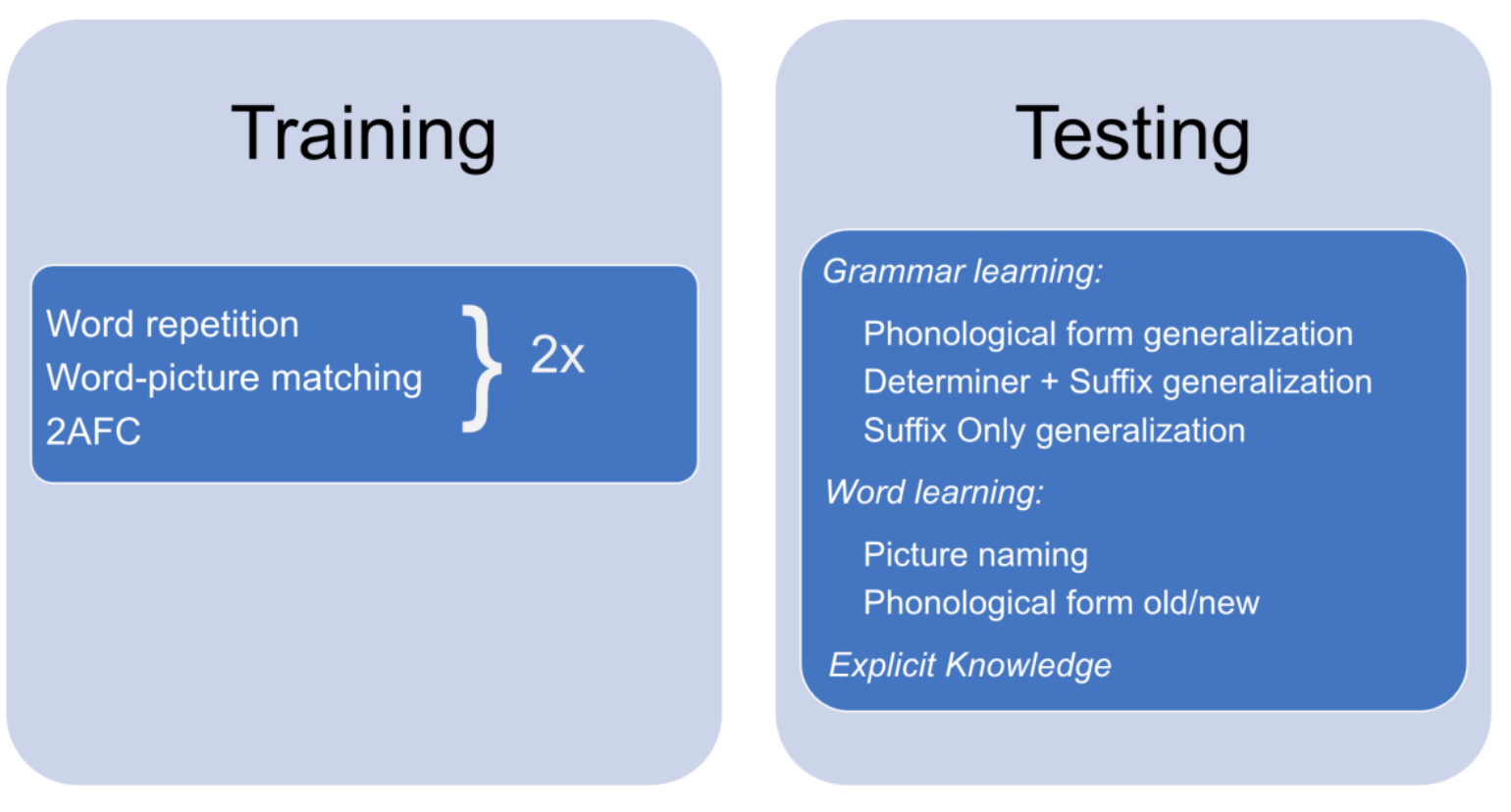

Figure 11. Experiment 3 design and tasks..

Training -- Word repetition. This new task was used to introduce a production component to the training procedure, and it was used as the first task at training. The same cover story about an alien coming to visit Earth was used as in Experiments 1 and 2. Each trial in this task started with the presentation of a fixation cross at the center of the screen for $500 \mathrm{~ms}$, followed by the presentation of the target image. The word was played over the speakers $500 \mathrm{~ms}$ after the image onset. The participant's task was to repeat the word out loud as they heard it. A spacebar press continued to the next trial. Each word was presented three times in total over two blocks, and the order of trials was randomized for each participant. There were two practice trials at the beginning of the task (using items that were not a part of the experimental manipulation). 
This task comprised the first training task in a cycle that consisted of Word repetition, Wordpicture matching, and the $2 \mathrm{AFC}$ task. The cycle was repeated twice. In total participants were exposed to each word-picture pair in this task 6 times.

Training -- Word-picture matching. This was the same task as in Experiments 1 and 2, but with only one exposure to each target pairing. In each cycle of this task there were 24 target (match) pairings, and 12 foil/mismatch pairings (counterbalanced for the mapping between the determiner and the suffix and the semantic category). Across the two cycles participants were exposed twice to each target word-picture pairing, and once to a mismatching pairing. Together, the training tasks lasted approximately 15 minutes.

Tests -- Word learning. Word learning was assessed using the 2AFC task during the training phase, and the Phonological form old/new judgement and Picture naming tasks at the end of the session. The 2AFC task had the same structure as in Experiments 1 and 2, and it assessed the learning of the meaning-form mappings for the newly learned words. The outcome measure was accuracy, as in Experiments 1 and 2.

The Phonological form old/new judgement task was used to assess the learning of the phonological form of the new words. Each trial in this task started with the presentation of a fixation cross at the center of the screen for $500 \mathrm{~ms}$, which was followed by a word played over the loudspeakers. The participant's task was to say out loud whether this was a word they had heard before. The experimenter wrote down the response on a response sheet (old ('heard before'), new ('not heard before')). There were 48 trials in total: 24 were the words from the training language, and 24 were foils. There were two lists of trials, with a fixed random order in each, and counterbalancing of the lists across participants. The outcome variable was accuracy on the old trials.

The Picture naming task was used to assess the learning of the form-meaning mapping in a language production task. Only the trained words were presented in this task, with a randomized order of presentation for each participant. Each trial in this task started with a presentation of the fixation cross at the center of the screen for $500 \mathrm{~ms}$, which was followed by the presentation of an image for a trained word. The image stayed on the screen for 6 seconds, or until the participant pressed the spacebar to proceed to the next trial. The responses were recorded by the experimenter, and coded for accuracy offline. Stems and affixes were coded separately. 
Tests -- Grammar learning. The same word-picture matching task as in Experiments 1 and 2 was used to assess generalization in the Determiner + Suffix and the Suffix Only tasks. In the new Phonological Form Only generalization task, each trial started with a presentation of the fixation cross for $500 \mathrm{~ms}$ which was followed by the audio presentation of a word item. As in the other generalization tasks, participants pressed the keys with a smiley or a sad face to indicate whether they thought the new word was a good word for the alien language. The order of presentation of the three generalization tasks (Determiner + Suffix, Suffix Only, Phonological Form Only) was counterbalanced across participants. A' was used as the outcome measure in all three tasks.

Explicit Knowledge questionnaire: We used the same two questions as in Experiments 1 and 2 to assess participants' explicit knowledge of the grammatical regularities. The test was administered after the experimental tasks, following a short break. The responses were coded as in Experiments 1 and 2.

\section{Data Analysis}

The data for the 2AFC task, the three generalization tasks, and the explicit knowledge questions were analyzed as in Experiments 1 and 2.

In the Phonological form old/new judgement task, we used one-tailed t-tests to compare accuracy against chances (.5) for each group. We also compared differences in performance between the two groups in a mixed-effects logistic regression with accuracy for the old trials coded as a binary outcome measure, and group as a fixed effect.

In the Picture naming task, we ran two separate mixed-effects logistic regression analyses. The first analysis used stem accuracy coded as a binary outcome measure and group as an effect coded fixed factor. The second used affix accuracy as a binary outcome measure, and group and affix as effect coded fixed factors (determiner $=.5$, suffix $=-.5$ ). In both analyses, trials where participants produced no responses were coded as 0 , and only fully accurate productions were coded as correct.

Results 


\section{Word learning}

At the end of training both children and adults showed similar levels of learning of the new words as in Experiment 2, as assessed by the 2AFC task (Figure 12; $\mathrm{M}_{\text {adults }}=.86, \mathrm{M}_{\text {children }}=.76$ ). Both groups performed significantly above chance (adults: $t(32)=16.16, p<.0001$; children: $t$ $(27)=9.81, p<.0001)$, and adults had overall better word learning than children $(b=.79, \mathrm{SE}=$ $.27, \mathrm{z}=2.94, \mathrm{p}=.003$ ). This finding confirms a beneficial effect of language production in vocabulary learning.

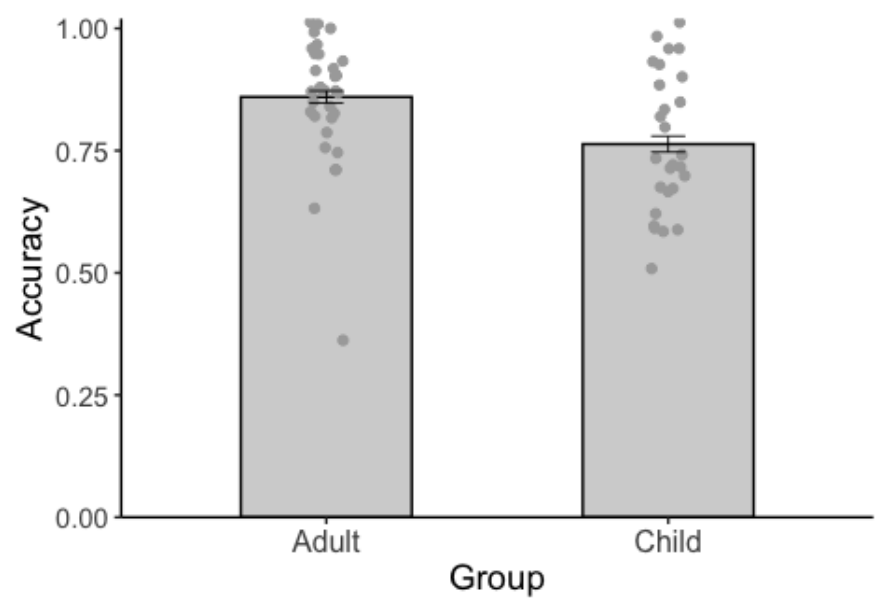

Figure 12. Accuracy on the 2AFC task measuring word learning.

In Experiment 3 we used two additional tests of word learning. The learning of the phonological form was assessed using the Phonological form old/new judgement task. As in the 2AFC task, here both groups performed significantly above chance (adults: $t(32)=16.38, p<.0001$; children: $t(27)=6.61, p<.0001)$, and the adults' learning of the phonological form was significantly better than the children's $(b=.98, S E=.22, z=4.54, p<.0001$, Figure 13).

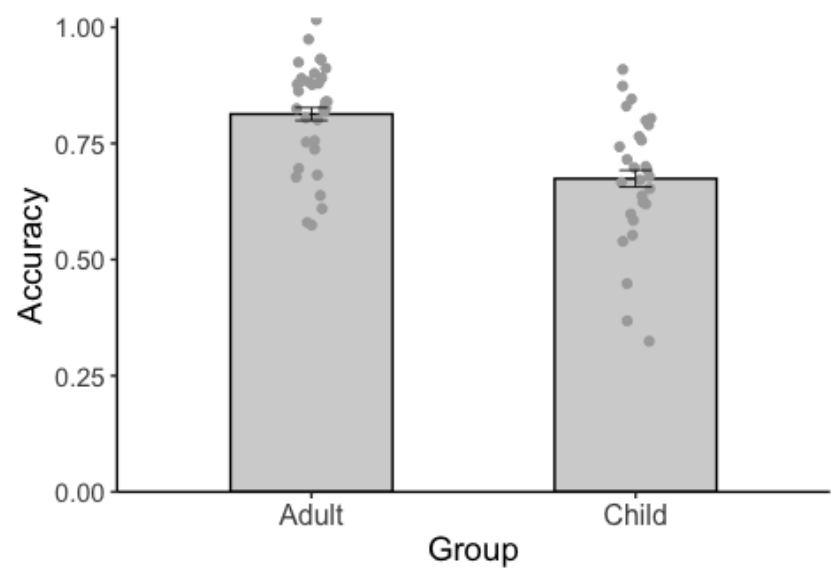


Figure 13. Accuracy on the Phonological Form Old/New task measuring the learning of the phonological form.

We also examined word learning using a picture naming task. This task allowed us to assess separately the learning of the stems (e.g., mof, larsh in mofeem, larshool), and of the affixes (tib, ked, eem, ool). We expected lower levels of accuracy in the stems due to the lower frequency of exposure relative to the two affixes (each unique word stem was presented 8 times during training, whereas the affixes were additionally repeated across all items within a grammatical category). The data were analyzed separately for the stems and the affixes. We examined group differences in both analyses, and we additionally examined the differences between the two affixes in the affix analysis.

The accuracy with the stems was low in both groups, but greater in adults than in children (Figure $14 ; \mathrm{b}=1.56, \mathrm{SE}=.51, \mathrm{z}=3.05, \mathrm{p}=.002$ ). The accuracy with the affixes was higher in both groups, and again greater in adults than in children (group in Table 11). In adults the accuracy was greater with the determiners than with the suffixes (group $\mathrm{x}$ affix interaction in Table 11; Figure 14). Overall, across all three elements of the new words and in both groups the accuracy was lower in this task relative to the two recognition-based tasks.
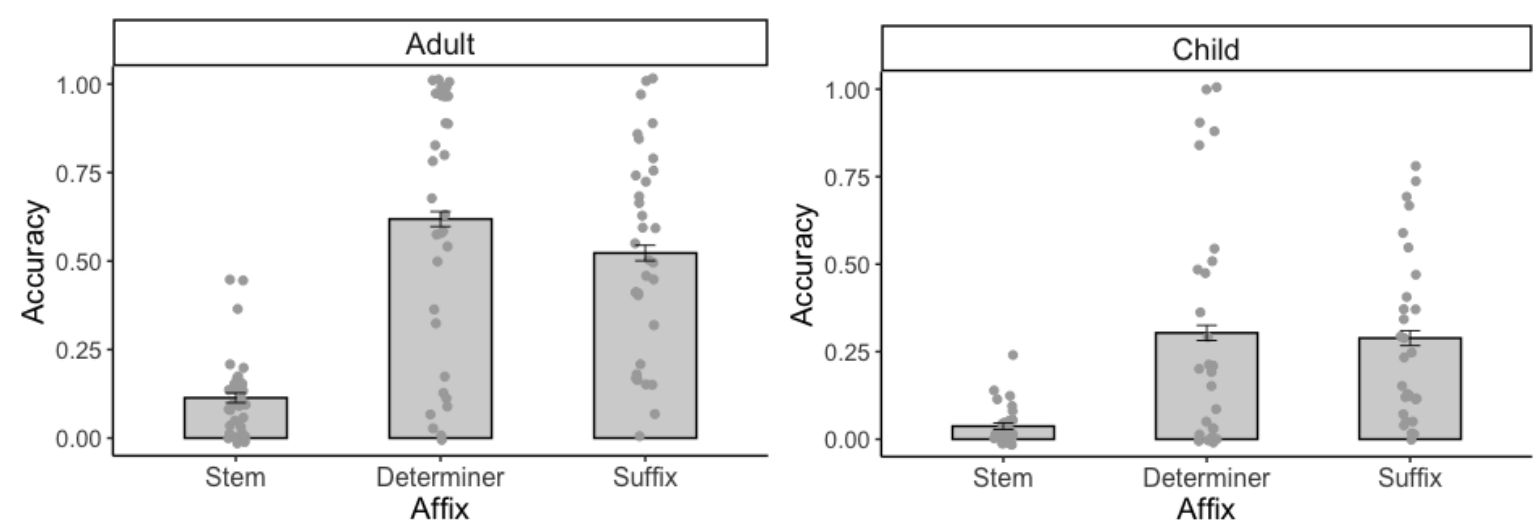

Figure 14. Accuracy on the Picture Naming task for the word stem, and the two affixes.

Table 11. Mixed effects regression coefficients for accuracy on the two affixes in the Picture naming task.

\begin{tabular}{lllll} 
& Estimate & Std. Error & $\mathrm{z}$ & $\mathrm{p}$ \\
\hline Group & 2.17 & .58 & 3.73 & $<.001$
\end{tabular}


Random effects included in the model: intercept by participants and item, slopes for affixes by participants, and group by items.

In sum, including a language production task in the training resulted in the same level of word learning as in Experiment 2, despite a decrease in the exposure to individual word-picture pairs. This finding confirms a beneficial effect of language production in vocabulary learning, i.e. the production effect. Across all three measures of word learning, and as in Experiments 1 and 2, adults had better performance than children. As expected, both children and adults showed better learning of the affixes than the stems (as evidenced in the picture naming task), and overall both groups showed better performance in recognition-based tasks (2AFC, Phonological form old/new judgements) than in the production-based task (Picture naming).

Use of grammatical regularities: Generalization tests

As in Experiments 1 and 2, we used generalization tests to assess the extent to which participants learned the grammatical regularities and were able to generalize them to previously unseen items. A key aim in Experiment 3 was to examine generalization performance when the number of exemplars was increased relative to Experiment 1, but the training regime did not include overexposure to individual word-picture pairs and included a language production element instead.

We first examined performance in the Determiner + Suffix generalization test. Now both adults and children showed evidence of generalization. In both groups a' scores were significantly above 0.5 (adults: $\mathrm{t}(32)=9.15, \mathrm{p}<.0001$ : children: $\mathrm{t}(27)=3.09, \mathrm{p}=.002$; Figure 15). Adults' a' scores were greater than the children's $(\mathrm{b}=.18, \mathrm{SE}=.07, \mathrm{t}=2.73, \mathrm{p}=.008)$. 

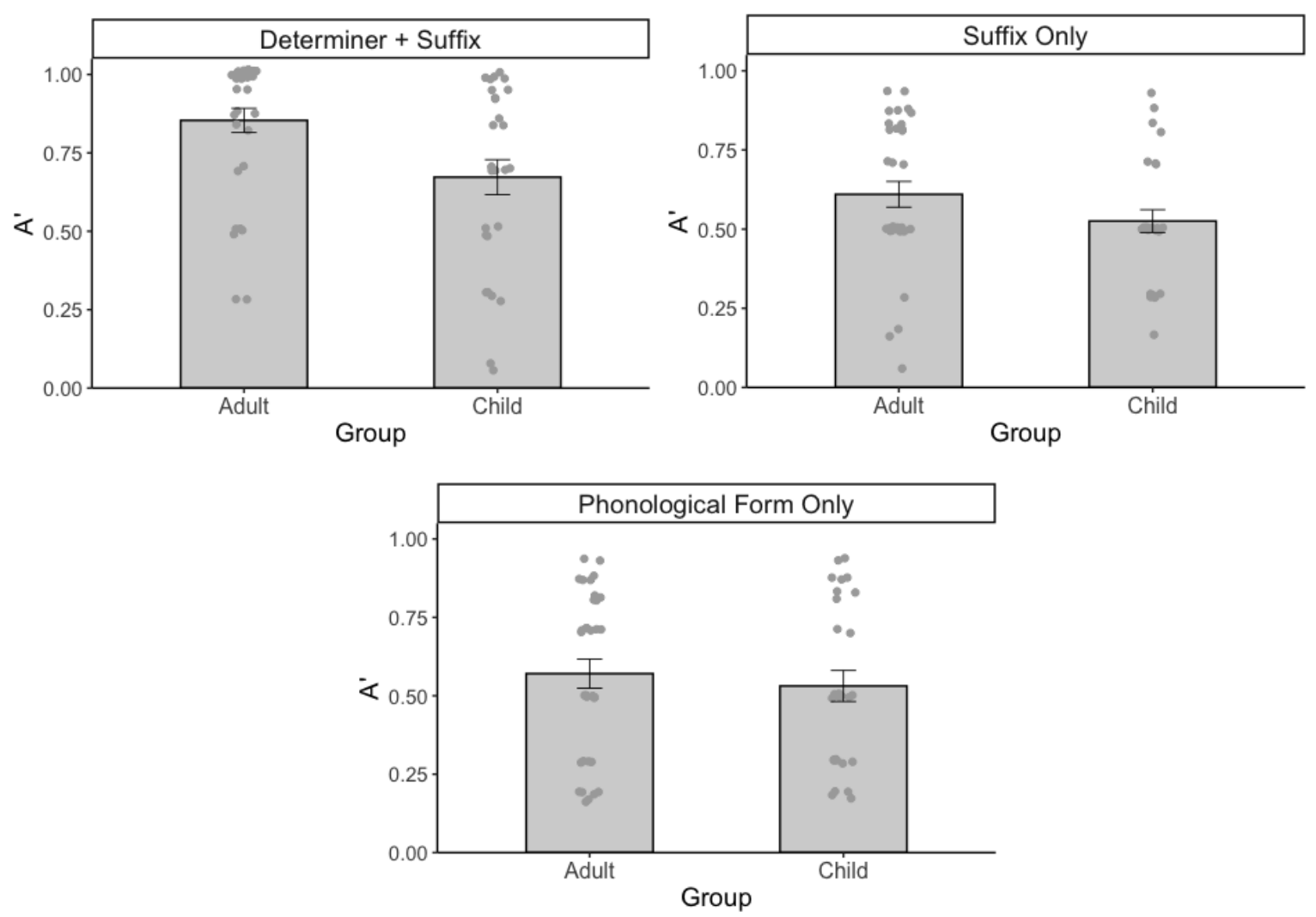

Figure 15. Performance on the three generalization tests for the two groups. Determiner + Suffix test assesses the regularity in the mapping between both affixes and the semantic properties of the referent; Suffix Only test assesses the co-occurrence between the suffix and the determiner, and the suffix and the semantic properties of the referent; Phonological Form Only test assess the co-occurrence in the determiner and the suffix only with no reference to semantic cues.

Next we examined performance in the Suffix Only generalization test. Adults' a' scores were now also greater than 0.5 , showing evidence of generalization in this task $(\mathrm{t}(32)=2.71, \mathrm{p}=.005$; Figure 15). Children did not show evidence of generalization ( $\mathrm{t}(27)=.70, \mathrm{p}=.244)$. The difference in a' scores between the groups was not reliable $(b=.09, \mathrm{SE}=.06, \mathrm{t}=1.53, \mathrm{p}=.13$ ).

Finally, we examined performance in the new Phonological Form Only generalization test. This test assessed the determiner-suffix distributional co-occurrence regularity (tib...eem, ked...ool) in the absence of semantic cues. There were no reliable differences from 0.5 in either group (adults: $t(32)=1.53, p=.07$; children: $t(27)=.63, p=.27$ ), showing no strong evidence of generalization in this test. There was no significant difference in a' scores between the two groups ( $\mathrm{b}=.04, \mathrm{SE}=.07, \mathrm{t}=.58, \mathrm{p}=.56)$.

In sum, including a language production component in the training and a greater number of exemplars (relative to Experiment 1) resulted in improved generalization in both children and 
adults. In Experiment 3 both adults and children successfully generalized in the Determiner + Suffix task, and adults additionally in the Suffix Only task. However, neither group showed evidence of generalization in the new Phonological Form Only task.

We examined next the extent to which explicit knowledge of the regularities emerged in the course of the session.

Explicit knowledge of affixation patterns, and its relationship to performance on the generalization tests

\section{Levels of explicit knowledge of affixes in children and adults}

Levels of explicit knowledge of the affixes were similar to those found in Experiment 1 (Figure 16). Adults again had overall greater explicit knowledge than children $(b=.94, S E=.19, t=4.87$, $\mathrm{p}<.0001$ ), both groups showed greater explicit knowledge of the determiners than the suffixes $(\mathrm{b}=1.29, \mathrm{SE}=.17, \mathrm{t}=7.55, \mathrm{p}<.0001)$, and this difference was more pronounced in adults than in children (group $x$ affix interaction: $b=.94, \mathrm{SE}=.34, \mathrm{t}=2.74, \mathrm{p}<.008$ ). These findings converge with Experiments 1 and 2, and demonstrate that explicit awareness of the regularities emerges in both groups in the course of training, and with significant individual variability in both groups.
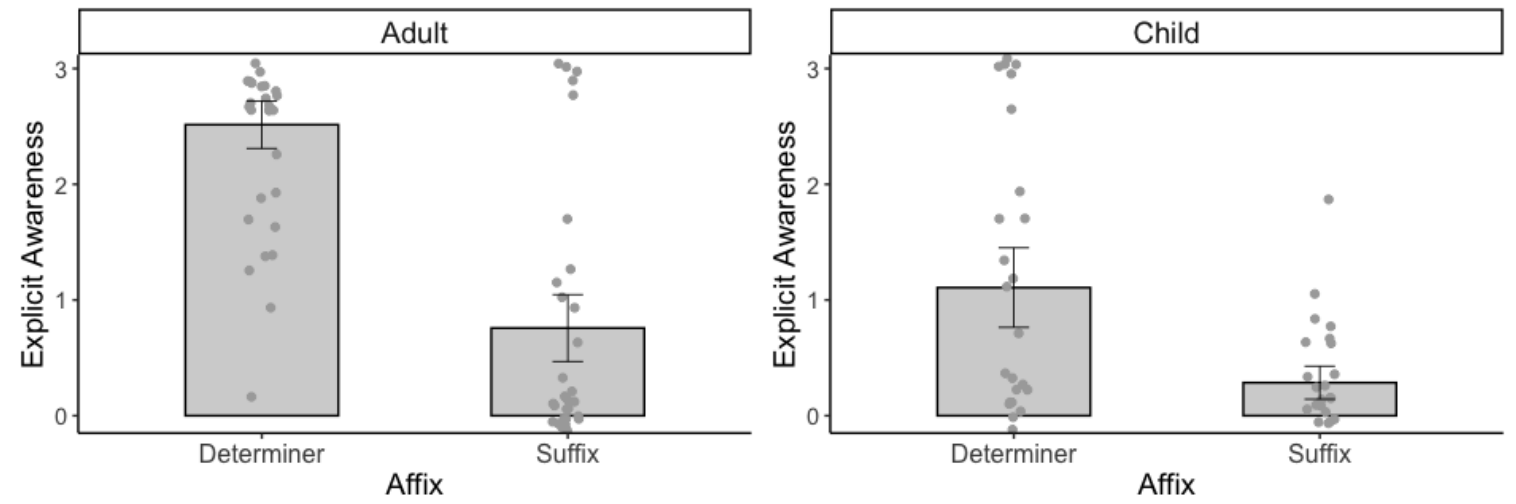

Figure 16. Levels of explicit knowledge of determiners and suffixes in adults and children.

Association between explicit knowledge of the regularities and performance on generalization tests 
We again ran linear regression analyses to examine the association between levels of explicit knowledge of the determiner and of the suffix and performance on the generalization tests. In Experiments 1 and 2 explicit knowledge was assessed in the delayed sessions, whereas in Experiment 3 it was assessed in the same session (as the very last task after all experimental tasks were completed).

A model predicting performance in the Determiner + Suffix generalization test including group and levels of explicit knowledge of the determiner and of the suffix accounted for $23 \%$ of the variance (total $\mathrm{r}^{2}=.23$, adjusted $\mathrm{r}^{2}=.19, \mathrm{~F}(3,57)=5.65, p=.002$ ). Both group and explicit knowledge of the determiner emerged as significant predictors, but explicit knowledge of the suffix did not (Table 12).

The models predicting performance in the Suffix Only and Phonological Form Only generalization tests accounted for non-significant proportions variance (Suffix Only: total ${ }^{2}$ $=.07$, adjusted $r^{2}=.04, \mathrm{~F}(2,58)=2.17, p=.124$; Phonological Form Only: total $\mathrm{r}^{2}=.006$, adjusted $\left.\mathrm{r}^{2}=-.03, \mathrm{~F}(2,58)=.18, p=.84\right)$. Neither group nor levels of explicit knowledge of the suffix predicted performance in these tasks (Table 12).

Table 12. Regression coefficients for Determiner + Suffix, Suffix Only, and Phonological Form Only generalization performance as the outcome variables, and group and explicit knowledge of affixes as predictors.

$$
\text { Determiner }+ \text { Suffix }
$$

Group

0.18

0.06

2.88

.006

Determiner knowledge

0.09

0.03

2.95

.005

Suffix knowledge

$-0.01$

.03

$-0.31$

.750

Suffix Only

Group

0.08

0.06

1.55

.130 
Suffix knowledge

Phonological Form Only

Group

Suffix knowledge
0.04

0.03

0.04

0.01
1.39

.169

\begin{tabular}{lllll} 
Suffix knowledge & 0.01 & 0.04 & 0.15 & .880 \\
\hline
\end{tabular}

In sum, with age group taken into account, successful performance in the Determiner + Suffix generalization test was predicted by participants' levels of explicit knowledge of the determiner. Neither age group nor levels of explicit knowledge of the suffix predicted performance in the Suffix Only test, in which adults but not children showed evidence of generalization. The performance in the Phonological Form Only generalization test was not predicted by age group or by levels of explicit knowledge of the suffix.

\section{Discussion}

In Experiment 3, we included a language production component at training and examined its effects on word learning and generalization of grammatical regularities. Participants in both groups showed similar levels of word learning as in Experiment 2, despite less exposure to individual word-picture pairs in the current experiment. This confirms previous findings of the benefits of language production in vocabulary learning in both children and adults (e.g., Hopman \& MacDonald, 2018; Icht \& Mama, 2015; Zamuner et al., 2016).

The inclusion of language production at training had additional beneficial effects for the learning and generalization of grammatical regularities. In adults, it resulted in successful generalization at the group level not only in the Determiner + Suffix test as in Experiments 1 and 2, but also in the Suffix Only test. In children, the inclusion of language production at training resulted in successful generalization in the Determiner + Suffix test for the first time. Interestingly, neither adults nor children showed evidence of generalization in the new Phonological Form Only generalization test. We turn to the variable levels of generalization in the different tests in General Discussion. 
As in Experiments 1 and 2, in both groups we found evidence of emergent explicit knowledge of the grammatical regularities that was greater in adults than in children. Explicit knowledge was greater for the more salient affix (determiner vs. suffix) in both groups, and this was more pronounced in adults than in children. However, it was only the explicit knowledge of the determiners that influenced performance on one of the generalization tests. Explicit knowledge of the suffix did not contribute to successful generalization in any of the tests. Together, these findings suggest that while explicit knowledge of the regularities plays a role in generalization, it is not necessary for successful generalization to occur.

\section{GENERAL DISCUSSION}

Across three experiments, we examined statistical learning of grammatical regularities in child and adult learners. Our aim was to address some of the limitations of the statistical learning literature identified in a recent review and meta-analysis by Frost and colleagues (Frost et al., 2019). First, in order to provide a more realistic representation of the learning environment, we used a paradigm that incorporated multiple regularities. We trained participants on an artificial language comprising phonological, distributional, and semantic regularities and mimicking grammatical gender systems in natural languages. Second, as natural language acquisition typically proceeds by simultaneous learning at several levels of linguistic structure, we implemented a paradigm that allowed us to examine grammar learning in the context of word learning, and including generalization of statistical regularities to untrained items. Third, in order to address the issue of underspecification of cognitive mechanisms underpinning statistical learning, we examined the role of off-line memory consolidation processes, and we systematically examined the emergence and contribution of explicit knowledge to generalization.

Across three experiments we showed that both children and adults learned the novel words well, but they had variable levels of success in generalizing the grammatical regularities (see Table 13 for a summary of findings). Generalization success depended on the age group, the type of regularity assessed, and the type of training. Adults successfully generalized in a greater range of conditions than the children. When assessing generalization in the test that included multiple regularities (Determiner + Suffix), the adults generalized well except in two conditions: their performance decreased when generalization was assessed a week after training (Experiment 1, Session 3), and when training included an enhanced exposure to individual words (Experiment 2). Children were generally poorer in generalization, and were successful in 
this test only when the training included a language production component (Experiment 3). Both adults and children were poorer or completely unsuccessful in generalizing the less salient regularities involving the suffix (Suffix Only), in particular in the absence of semantic cues (Phonological Form Only generalization in Experiment 3). The unsuccessful generalization of the distributional co-occurrence regularities (tib...eem, ked...ool) in the absence of semantic cues is particularly intriguing in the context of previous evidence of the ability of even 15-month old infants to learn this type of regularity when it is implemented as the only statistical regularity in the language (e.g., Gómez \& Maye, 2005). Thus the variability in generalization patterns for different types of regularities in both age groups in the current study crucially demonstrates the importance of assessing statistical learning using learning environments that are more representative of the complexities of natural languages.

The inclusion of additional exemplars in the training set (e.g., Gómez, 2002) was helpful for generalization only when language production was used at training (Experiment 3), but not when the training task included an enhanced exposure to individual words (Experiment 2). The use of language production at training was beneficial in both age groups, and for both word and grammar learning. The benefit of language production in word learning is consistent with the previous evidence of the production effect (e.g., Hopman \& MacDonald, 2018; Icht \& Mama, 2015; MacLeod \& Bodner, 2017; Zamuner et al., 2016). Perhaps more interestingly, the involvement of the language production system at training resulted in a beneficial effect for grammar learning. This benefit was shown in adults' successful generalization with the less salient suffix regularity (in the Suffix Only test) only when language production was engaged at training (Experiment 3). Likewise, children successfully generalized in the Determiner + Suffix test only when required to repeat the words at training (Experiment 3). As hypothesized by Hopman and MacDonald (2018), these findings suggest that the utterance planning processes including binding across different levels of linguistic representations facilitate the learning of the statistical regularities involving co-dependencies between different elements of the novel words (e.g., tib + eem, ked + ool; eem + animals, ool + artifacts).

Table 13: Word learning and generalization of grammatical regularities.

Experiment 1
Experiment 2

Experiment 3

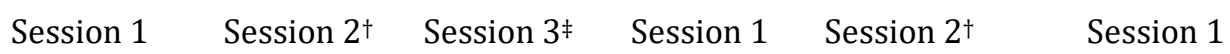

\section{Word learning}




\begin{tabular}{|c|c|c|c|c|c|c|c|}
\hline \multirow{2}{*}{$\begin{array}{l}2 \mathrm{AFC}: \\
\text { proportion corre }\end{array}$} & adult & $.98 * * *$ & $.88^{* * *}$ & $.86^{* * *}$ & $.84^{* * *}$ & $.86^{* * *}$ & $.86^{* * *}$ \\
\hline & child & $.75^{* * *}$ & $.73^{* * *}$ & $.70^{* * *}$ & $.75^{* * *}$ & $.75^{* * *}$ & $.76^{* * *}$ \\
\hline \multicolumn{8}{|l|}{ Generalization } \\
\hline \multirow{3}{*}{$\begin{array}{l}\text { Determiner + } \\
\text { Suffix a' }\end{array}$} & adult & $.78^{* * *}$ & $.79 * * *$ & .59 & .54 & $.65^{*}$ & $.85^{* * *}$ \\
\hline & & & & & & & \\
\hline & child & .44 & .43 & .45 & .48 & .46 & $.67^{* *}$ \\
\hline \multirow[t]{2}{*}{ Suffix Only a' } & adult & .55 & .57 & .43 & .58 & .50 & $.61^{* *}$ \\
\hline & child & .52 & .53 & $.62^{*}$ & .51 & .49 & .53 \\
\hline \multirow{3}{*}{$\begin{array}{l}\text { Phonological } \\
\text { Form Only a' }\end{array}$} & adult & - & - & - & - & - & .57 \\
\hline & & & & & & & \\
\hline & child & - & - & - & - & - & .53 \\
\hline
\end{tabular}

†Session 2 was run approximately 24 hours after Session 1. ‡Session 3 was run approximately 1 week after Session 1.

t-tests against $0.5:{ }^{* *} \mathrm{p}<.001 ;{ }^{* *} \mathrm{p}<.01,{ }^{*} \mathrm{p}<.05$

In order to shed more light on the cognitive mechanisms underpinning statistical learning, we examined the role of off-line memory consolidation processes. After training, both child and adult learners were tested immediately (all experiments), and after short (approximately $24 \mathrm{hr}$ ) (Experiments 1 and 2), and long (approximately 1 week) delays (Experiment 1). Overall, children were minimally influenced by the delays between training and testing. In word learning, even though there was some evidence of a decrease in performance over time, children's novel word knowledge was remarkably stable across both short and long delays (Table 13, Experiments 1 and 2). These findings converge with evidence for the role of off-line consolidation processes in the maintenance of lexical knowledge in this age group (e.g., Brown et al., 2012; Henderson et al., 2012, 2015). Children's generalization performance was only weakly influenced by the delays. A day-long off-line period did not change their performance in either of the generalization tests (Session 2 in Experiments 1 and 2), and a week-long delay 
slightly improved the performance in only one of the tests (Suffix Only in Session 3, Experiment 1).

Adults were influenced by off-line consolidation processes in a different way than children. In word learning, there was a significantly greater drop in performance after a day-long delay relative to children (10\% in adults vs. $2 \%$ in children), suggesting stronger maintenance of the newly learned words in children over short delays (e.g., Bishop et al., 2012; Weighall et al., 2017). Importantly, and similar to children, there was good maintenance of the newly acquired lexical knowledge over a week-long delay (accuracy at 86\% after a week-long delay; Table 13). The findings were less consistent in the tests of generalization. When it was already successful immediately after learning (Determiner + Suffix test in Experiment 1), a short delay did not change the adults' generalization performance, but a week-long delay led to a decrease in successful generalization. When generalization was not successful immediately after learning, in the majority of cases the delays did not lead to a change in performance (Sessions 2 and 3 in Experiment 1, and Session 2 in Experiment 2, for the Suffix Only test). The only exception was a day-long delay condition in Experiment 2 for the Determiner + Suffix test. Together, these findings provide further evidence for the role of off-line consolidation processes in the long term maintenance of lexical knowledge in both adults and children (e.g., Davis \& Gaskell, 2009; James et al., 2017). Future studies are needed to further examine the conditions in which grammatical generalizations may be facilitated vs. hindered by off-line memory consolidation processes. The current findings highlight the importance of assessing performance in statistical learning studies both immediately after learning and after a delay to examine the contribution of domain-general memory consolidation processes to the longevity of statistical learning.

To further examine the cognitive mechanisms underpinning statistical learning, in all three experiments we assessed the extent to which explicit knowledge of the regularities emerged in the course of the study. To reduce a possible role of the test itself influencing performance on the experimental tasks (e.g., Monaghan et al., 2019), explicit knowledge was always assessed after the experimental tasks were completed. This meant that explicit knowledge was assessed immediately after training (Experiment 3), or after a short or a long delay (Experiments 2 an $\mathrm{d} 1$ respectively). We found evidence of emergent explicit knowledge in both children and adults immediately after training (Experiment 3), and this knowledge was maintained over a day-long (Experiments 1 and 2) and a week-long delay (Experiment 1) (see Table 14 for a summary). In all three experiments, adults showed evidence of greater explicit knowledge than children. This could be partly due to the nature of our measure requiring an explicit verbal report, and thus being more demanding for the children. In both groups we found greater levels of explicit 
knowledge of the regularities involving the determiners than the regularities involving the suffixes.

Table 14: Means (standard deviations) for levels of explicit knowledge emerging in the course of the experiment (maximum score $=3$ ).

Experiment 1

Experiment 2

Experiment 3

\begin{tabular}{|c|c|c|c|c|c|c|c|}
\hline & & Session 1 & Session $2^{+}$ & Session $3^{\ddagger}$ & Session 1 & Session $2^{\dagger}$ & Session 1 \\
\hline \multirow[t]{2}{*}{ Determiners } & adult & - & - & $2.61(0.70)$ & - & $1.52(0.93)$ & 2.52 (1.18) \\
\hline & child & - & - & $0.65(1.04)$ & - & $0.43(0.10)$ & $1.11(1.82)$ \\
\hline \multirow[t]{2}{*}{ Suffixes } & adult & - & - & $0.28(0.75)$ & - & $0.04(0.93)$ & $0.76(1.66)$ \\
\hline & child & - & - & $0.10(0.31)$ & - & $0.03(0.10)$ & $0.29(0.76)$ \\
\hline
\end{tabular}

†Session 2 was run approximately 24 hours after Session 1 . ‡Session 3 was run approximately 1 week after Session 1. Explicit knowledge was assessed at Session 3 in Experiment 1, at Session 2 in Experiment 2, and at Session 1 in Experiment 3.

The contribution of the emergent explicit knowledge to generalization was assessed in a series of regression analyses while taking age group into account (Table 15). Explicit knowledge of the determiners contributed positively to generalization performance in all three experiments in the Determiner + Suffix test. Interestingly, in Experiment 1 generalization performance in this test was significantly lower at a week-delayed session (Table 13) despite high levels of explicit knowledge of the determiners during the same session (and a marginally significant contribution to generalization performance $(\mathrm{p}=.08))$. The contribution of explicit knowledge of the suffixes was inconsistent across the different tasks: it did not contribute to either the Suffix Only or the Phonological Form Only tests in any of the experiments, and it contributed negatively to the Determiner + Suffix generalization performance in Experiment 1. These findings suggest that while emergent explicit knowledge contributes to the generalization of grammatical regularities, it is not the key driver of successful generalization. Together with the findings from the studies of statistical learning of phonological regularities (Batterink et al., 
2015; Smalle et al., 2018), these findings provide converging evidence for the role of explicit knowledge in statistical learning tasks, in this case in grammatical generalization.

Table 15: Regression analyses with performance at the three generalization tests as the outcome measures.

\begin{tabular}{|c|c|c|c|c|c|c|}
\hline & Session 1 & Session $2^{+}$ & Session $3^{\ddagger}$ & Session 1 & Session $2^{+}$ & Session 1 \\
\hline \multicolumn{7}{|l|}{ Determiner + Suffix test } \\
\hline Group & $.34^{* * *}$ & $.32^{* *}$ & .08 & .05 & $.19^{*}$ & $.18^{* *}$ \\
\hline Determiner knowledge & $.13^{*}$ & .05 & $.13^{\S}$ & .04 & $.10^{*}$ & $.09 * *$ \\
\hline Suffix knowledge & $-.19 *$ & $-.17 \S$ & .11 & .07 & -.01 & -.01 \\
\hline \multicolumn{7}{|l|}{ Suffix Only test } \\
\hline Group & .07 & .06 & $-.20 \S$ & .06 & .01 & .08 \\
\hline Suffix knowledge & -.07 & .11 & -.05 & -.05 & .01 & .04 \\
\hline \multicolumn{7}{|l|}{ Phonological Form Only test } \\
\hline Group & - & - & - & - & - & .04 \\
\hline Suffix knowledge & - & - & - & - & - & .01 \\
\hline
\end{tabular}

†Session 2 was run approximately 24 hours after Session 1. ¥Session 3 was run approximately 1 week after Session 1. Explicit knowledge was assessed at Session 3 in Experiment 1, at Session 2 in Experiment 2, and at Session 1 in Experiment 3.

*** $\mathrm{p}<.001 ;{ }^{* *} \mathrm{p}<.01,{ }^{*} \mathrm{p}<.05$, sp $<.10$

Implications for theories of statistical learning in language development

Our findings have three key implications for theories of statistical learning and its role in language development. 
First, we have shown that realistic learning environments that incorporate multiple regularities result in different patterns of generalization than simpler learning environments that have been used in the majority of previous statistical learning studies (e.g., Gerken, 2006; Gerken et al., 2005; Gómez, 2002; Gómez et al., 2006; Gómez \& Gerken, 1999; Gómez \& Lakusta, 2004; Gómez \& Maye, 2005; Reeder et al., 2013, 2017). In our paradigm, which incorporates three types of regularities that have independently been shown to play a role in grammar learning, both child and adult learners show generalizations that prioritize regularities that involve semantic cues. Prioritization of semantic regularities over distributional cues when learning form-to-meaning mappings may be due to the nature of the training task we used that focused on learning word meanings, a key function of language and communication. This is unlike many other statistical learning studies that typically use simple exposure to isolated uni-dimensional regularities, and often use grammaticality judgement or classifications tasks to assess the learning (e.g., Gerken, 2006; Gómez, 2002; Gómez \& Lakusta, 2004; Reeder et al., 2013, 2017). More broadly, our findings are consistent with other evidence in statistical learning in different domains that the structure and complexity of the input act as a constraint on statistical learning in that they shape what regularites are learned (Fiser \& Aslin, 2005; Plaut \& Vande Velde, 2017; Romberg \& Saffran, 2013; Turk-Browne et al., 2008). We believe that together these data show convincingly that using learning environments that are more representative of the real learning situation is crucial for future studies and theories of statistical learning (see Siegelman et al., 2020 for a related demonstration in literacy acquisition).

Second, our findings provide further evidence in support of multicomponential views of statistical learning (e.g., Conway, 2020; Frost et al., 2019). According to these views, statistical learning is a domain-general learning mechanism in that it reflects a general property of neural plasticity that is instantiated in similar computational principles across a range of neural networks supporting it. These approaches further propose that both domain-specific and domain-general neural regions underpin statistical learning, including areas traditionally associated with declarative/explicit and procedural/implicit memory systems (Batterink et al., 2019). Conway (2020) specifically focuses on two key neocortical systems: first, a posterior, modality-specific system involved in encoding both modality-specific regularities over shorter timescales, and more complex regularities across domains over longer timescales; second, a fronto-parietal system mediating modulatory effects of attention and model-based aspects of learning. The two systems are proposed to work in parallel, in some situations cooperatively, and in others competitively (e.g., Ambrus et al., 2020; Batterink et al., 2019). Additionally, subcortical structures traditionally involved in declarative and procedural memory (e.g., medial 
temporal lobe and the hippocampus, and basal ganglia respectively) are thought to be involved in the long-term maintenance of the acquired knowledge (Batterink et al., 2019; Conway, 2020; Frost et al., 2015). Our findings involving a learning environment with multiple regularities, and including contributions of explicit knowledge to generalization, are more easily accommodated within a multicomponential view of statistical learning. Within this framework, learning and generalization of multi-modal (visual, auditory) and multi-domain (phonological, semantic) regularities is likely to involve more anterior regions of the posterior cortical component in the model proposed by Conway, and the contribution of explicit knowledge may be more likely to engage the areas of the frontal cortex (e.g., van Kesteren et al., 2012). Additionally, the evidence of the changes in generalization performance across the delay periods supports the proposals that areas associated with consolidation and maintenance of knowledge (e.g., MTL) might be involved.

Finally, only a small minority of traditional statistical learning studies have examined the learning outside of a narrow range of early childhood, and when using adults the assumption has been that the same unitary implicit learning mechanism present in infants underpins the learning. However, a recent body of evidence suggests that linguistic representations continue to be shaped by language environment throughout the lifespan (see Seidenberg \& MacDonald, 2018 for a review). Literacy is one of the most well examined areas in this context (e.g., Arciuli \& Simpson, 2012; Siegelman et al., 2020; Spencer et al., 2015; Ulicheva et al., 2020; see Sawi \& Rueckl, 2019 for a review), but language environment shapes linguistic representations from speech sounds (e.g., Idemaru \& Holt, 2011, 2020; Lehet \& Holt, 2017) to syntactic structures and discourse (e.g., Amato \& MacDonald, 2010; Arnold et al., 2018; Arnold et al, 2019; Haskell et al., 2010; Langlois \& Arnold, 2020; Mirković \& MacDonald, 2013; Montag \& MacDonald, 2015; Wells et al., 2009). Within a multicomponential view of statistical learning, these effects may be underpinned by different contributing processes with different developmental trajectories. Thus, beyond including more realistic learning environments, future studies should also expand the age range across which statistical learning is examined and might contribute to language development.

\section{Acknowledgments}

We would like to thank William Cook, Jessica Innes, and Samantha Stansfield for their help with data collection, and Amanda Hickey for useful discussions. 


\section{CRediT statement}

J. Mirković: Conceptualization; Methodology; Formal analysis; Data curation; Writing-original draft; Writing-review \& editing; Supervision. M. Yee: Methodology; Investigation; Writing original draft. M. Kennedy: Investigation; Writing-original draft. M. E. Hayiou-Thomas: Conceptualization; Methodology; Writing-original draft; Writing - review \& editing; Supervision. 


\section{References}

Amato, M. S., \& MacDonald, M. C. (2010). Sentence processing in an artificial language: Learning and using combinatorial constraints. Cognition, 116(1), 143-148.

Amso, D., \& Davidow, J. (2012). The development of implicit learning from infancy to adulthood: item frequencies, relations, and cognitive flexibility. Developmental Psychobiology, 54(6), 664-673.

Arciuli, J., \& Simpson, I. C. (2012). Statistical learning is related to reading ability in children and adults. Cognitive Science, 36(2), 286-304.

Arnold, J. E., Castro-Schilo, L., Zerkle, S., \& Rao, L. (2019). Print exposure predicts pronoun comprehension strategies in children. Journal of Child Language, 1-31.

Arnold, J. E., Strangmann, I. M., Hwang, H., Zerkle, S., \& Nappa, R. (2018). Linguistic experience affects pronoun interpretation. Journal of Memory and Language, 102, 41-54.

Aslin, R. N. (2017). Statistical learning: a powerful mechanism that operates by mere exposure. Wiley Interdisciplinary Reviews. Cognitive Science, 8(1-2). https://doi.org/10.1002/wcs.1373

Aslin, R. N., \& Newport, E. L. (2012). Statistical learning: From acquiring specific items to forming general rules. Current Directions in Psychological Science, 21(3), 170-176.

Barr, D. J., Levy, R., Scheepers, C., \& Tily, H. J. (2013). Random effects structure for confirmatory hypothesis testing: Keep it maximal. Journal of Memory and Language, 68(3). https://doi.org/10.1016/j.jml.2012.11.001

Bates, D., Mächler, M., Bolker, B., \& Walker, S. (2015). Fitting Linear Mixed-Effects Models Using lme4. In Journal of Statistical Software (Vol. 67, Issue 1, pp. 1-48). https://doi.org/10.18637/jss.v067.i01

Bates, E., \& Goodman, J. C. (1997). On the inseparability of grammar and the lexicon: Evidence from acquisition, aphasia and real-time processing. Language and Cognitive Processes, 12(56), 507-584.

Batterink, L. J., Paller, K. A., \& Reber, P. J. (2019). Understanding the neural bases of implicit and statistical learning. Topics in Cognitive Science, 11(3), 482-503.

Batterink, L. J., Reber, P. J., Neville, H. J., \& Paller, K. A. (2015). Implicit and explicit contributions to statistical learning. Journal of Memory and Language, 83, 62-78.

Berko, J. (1958). The Child's Learning of English Morphology. Word \& World, 14(2-3), 150-177.

Bishop, D. V. M., Barry, J. G., \& Hardiman, M. J. (2012). Delayed retention of new word-forms is better in children than adults regardless of language ability: a factorial two-way study. PloS One, 7(5), e37326.

Boyd, J. K., \& Goldberg, A. E. (2012). Young children fail to fully generalize a novel argument 
structure construction when exposed to the same input as older learners. Journal of Child Language, 39(3), 457-481.

Brown, H., Weighall, A., Henderson, L., \& Gaskell, G. M. (2012). Enhanced recognition and recall of new words in 7- and 12-year-olds following a period of offline consolidation. Journal of Experimental Child Psychology, 112(1), 56-72.

Cassidy, K. W., \& Kelly, M. H. (1991). Phonological information for grammatical category assignment. Journal of Memory and Language, 30, 348-369.

Cassidy, K. W., \& Kelly, M. H. (2001). Children's use of phonology to infer grammatical class in vocabulary learning. Psychonomic Bulletin \& Review, 8, 519-523.

Christiansen, M. H. (2019). Implicit Statistical Learning: A Tale of Two Literatures. Topics in Cognitive Science, 11(3), 468-481.

Conway, C. M. (2020). How does the brain learn environmental structure? Ten core principles for understanding the neurocognitive mechanisms of statistical learning. Neuroscience and Biobehavioral Reviews, 112, 279-299.

Corbett, G. G. (1991). Gender. Cambridge University Press.

Culbertson, J., Gagliardi, A., \& Smith, K. (2017). Competition between phonological and semantic cues in noun class learning. Journal of Memory and Language, 92, 343-358.

Davis, M. H., \& Gaskell, M. G. (2009). A complementary systems account of word learning: neural and behavioural evidence. Philosophical Transactions of the Royal Society of London. Series B, Biological Sciences, 364(1536), 3773-3800.

Dikker, S., Rabagliati, H., Farmer, T. A., \& Pylkkänen, L. (2010). Early occipital sensitivity to syntactic category is based on form typicality. Psychological Science, 21(5), 629-634.

Dobel, C., Junghöfer, M., Breitenstein, C., Klauke, B., Knecht, S., Pantev, C., \& Zwitserlood, P. (2010). New names for known things: On the association of novel word forms with existing semantic information. Journal of Cognitive Neuroscience, 22, 1251-1261.

Farmer, T. A., Christiansen, M. H., \& Monaghan, P. (2006). Phonological typicality influences online sentence comprehension. Proceedings of the National Academy of Sciences, 103, 12203.

Finn, A. S., Kalra, P. B., Goetz, C., Leonard, J. A., Sheridan, M. A., \& Gabrieli, J. D. E. (2016). Developmental dissociation between the maturation of procedural memory and declarative memory. Journal of Experimental Child Psychology, 142, 212-220.

Fiser, J., \& Aslin, R. N. (2005). Encoding multielement scenes: statistical learning of visual feature hierarchies. Journal of Experimental Psychology. General, 134(4), 521-537.

Forster, K. I., \& Forster, J. C. (2003). DMDX: a windows display program with millisecond accuracy. Behavior Research Methods, Instruments, \& Computers: A Journal of the Psychonomic Society, Inc, 35(1), 116-124.

Franco, A., Cleeremans, A., \& Destrebecqz, A. (2011). Statistical learning of two artificial 
languages presented successively: how conscious? Frontiers in Psychology, 2, 229.

Frigo, L., \& McDonald, J. L. (1998). Properties of phonological markers that affect the acquisition of gender-like subclasses. Journal of Memory and Language, 39, 218-245.

Frost, R., Armstrong, B. C., \& Christiansen, M. H. (2019). Statistical learning research: A critical review and possible new directions. Psychological Bulletin, 145(12), 1128-1153.

Frost, R., Armstrong, B. C., Siegelman, N., \& Christiansen, M. H. (2015). Domain generality versus modality specificity: the paradox of statistical learning. Trends in Cognitive Sciences, 19(3), 117-125.

Frost, R. L. A., \& Monaghan, P. (2016). Simultaneous segmentation and generalisation of nonadjacent dependencies from continuous speech. Cognition, 147, 70-74.

Gentner, D. (1982). Why nouns are learned before verbs: linguistic relativity versus natural partitioning. In S. A. Kuczaj (Ed.), Language development: Vol.2. Language, thought and culture (pp. 301-334). Lawrence Erlbaum Associates.

Gerken, L. (2006). Decisions, decisions: infant language learning when multiple generalizations are possible. Cognition, 98(3), B67-B74.

Gerken, L. (2010). Infants use rational decision criteria for choosing among models of their input. Cognition, 115(2), 362-366.

Gerken, L., Wilson, R., \& Lewis, W. (2005). Infants can use distributional cues to form syntactic categories. Journal of Child Language, 32, 249-268.

Gómez, R. L. (2002). Variability and detection of invariant structure. Psychological Science, 13, 431-436.

Gómez, R. L., Bootzin, R. R., \& Nadel, L. (2006). Naps promote abstraction in language-learning infants. Psychological Science, 17(8), 670-674.

Gómez, R. L., \& Gerken, L. (1999). Artificial grammar learning by 1-year-olds leads to specific and abstract knowledge. Cognition, 70(2), 109-135.

Gómez, R. L., \& Gerken, L. (2000). Infant artificial language learning and language acquisition. Trends in Cognitive Sciences, 4(5), 178-186.

Gómez, R. L., \& Lakusta, L. (2004). A first step in form-based category abstraction by 12-monthold infants. Developmental Science, 7(5), 567-580.

Gómez, R. L., \& Maye, J. (2005). The Developmental Trajectory of Nonadjacent Dependency Learning. Infancy: The Official Journal of the International Society on Infant Studies, 7(2), 183-206.

Haskell, T., Thornton, R., \& MacDonald, M. C. (2010). Experience and grammatical agreement: Statistical learning shapes number agreement production. Cognition, 114, 151-164.

Henderson, L., Devine, K., Weighall, A., \& Gaskell, G. M. (2015). When the daffodat flew to the intergalactic zoo: off-line consolidation is critical for word learning from stories. 
Developmental Psychology, 51(3), 406-417.

Henderson, L., Weighall, A. R., Brown, H., \& Gaskell, G. M. (2012). Consolidation of vocabulary is associated with sleep in children. Developmental Science, 15(5), 674-687.

Hopman, E. W. M., \& MacDonald, M. C. (2018). Production Practice During Language Learning Improves Comprehension. Psychological Science, 956797618754486.

Hupbach, A., Gómez, R. L., Bootzin, R. R., \& Nadel, L. (2009). Nap-dependent learning in infants. Developmental Science, 12(6), 1007-1012.

Icht, M., \& Mama, Y. (2015). The production effect in memory: a prominent mnemonic in children. Journal of Child Language, 42(5), 1102-1124.

Idemaru, K., \& Holt, L. L. (2011). Word recognition reflects dimension-based statistical learning. Journal of Experimental Psychology. Human Perception and Performance, 37(6), 1939-1956.

Idemaru, K., \& Holt, L. L. (2020). Generalization of dimension-based statistical learning. Attention, Perception \& Psychophysics, 82(4), 1744-1762.

James, E., Gaskell, M. G., Weighall, A., \& Henderson, L. (2017). Consolidation of vocabulary during sleep: The rich get richer? Neuroscience and Biobehavioral Reviews, 77, 1-13.

Karpicke, J. D., \& Roediger, H. L., 3rd. (2008). The critical importance of retrieval for learning. Science, 319(5865), 966-968.

Kelly, M. H. (1992). Using sound to solve syntactic problems: The role of phonology in grammatical category assignments. Psychological Review, 99, 349-364.

Kidd, E. (2012). Implicit statistical learning is directly associated with the acquisition of syntax. Developmental Psychology, 48(1), 171-184.

Langlois, V. J., \& Arnold, J. E. (2020). Print exposure explains individual differences in using syntactic but not semantic cues for pronoun comprehension. Cognition, 197, 104155.

Lany, J., \& Saffran, J. R. (2010). From statistics to meaning: infants' acquisition of lexical categories. Psychological Science, 21(2), 284-291.

Lany, J., \& Saffran, J. R. (2011). Interactions between statistical and semantic information in infant language development. Developmental Science, 14(5), 1207-1219.

Lany, J., \& Saffran, J. R. (2013). Statistical learning mechanisms in infancy. In J. Rubenstein \& P. Rakic (Eds.), Neural Circuit Development and Function in the Healthy and Diseased Brain. Academic Press.

Lehet, M., \& Holt, L. L. (2017). Dimension-Based Statistical Learning Affects Both Speech Perception and Production. Cognitive Science, 41 Suppl 4, 885-912.

MacLeod, C. M., \& Bodner, G. E. (2017). The Production Effect in Memory. Current Directions in Psychological Science, 26(4), 390-395.

McDonough, C., Song, L., Hirsh-Pasek, K., Golinkoff, R. M., \& Lannon, R. (2011). An image is worth a thousand words: why nouns tend to dominate verbs in early word learning. 
Developmental Science, 14(2), 181-189.

Mirković, J., Forrest, S., \& Gaskell, M. G. (2011). Semantic regularities in grammatical categories: Learning grammatical gender in an artificial language. Proceedings of the Annual Meeting of the Cognitive Science Society, 33, 324-329.

Mirković, J., \& Gaskell, M. G. (2016). Does sleep improve your grammar? Preferential consolidation of arbitrary components of new linguistic knowledge. PloS One, 11(4), e0152489.

Mirković, J., \& MacDonald, M. C. (2013). When singular and plural are both grammatical: Semantic and morphophonological effects in agreement. Journal of Memory and Language, 69(3), 277-298.

Mirković, J., MacDonald, M. C., \& Seidenberg, M. S. (2005). Where does gender come from? Evidence from a complex inflectional system. Language and Cognitive Processes, 20, 139167.

Mirković, J., Vinals, L., \& Gaskell, M. G. (2019). The role of complementary learning systems in learning and consolidation in a quasi-regular domain. Cortex, 116, 228-249.

Monaghan, P., Chater, N., \& Christiansen, M. H. (2005). The differential role of phonological and distributional cues in grammatical categorisation. Cognition, 96, 143-182.

Monaghan, P., Christiansen, M. H., \& Chater, N. (2007). The phonological-distributional coherence hypothesis: Cross-linguistic evidence in language acquisition. Cognitive Psychology, 55, 259-305.

Monaghan, P., Schoetensack, C., \& Rebuschat, P. (2019). A Single Paradigm for Implicit and Statistical Learning. Topics in Cognitive Science, 11(3), 536-554.

Montag, J. L., \& MacDonald, M. C. (2015). Text exposure predicts spoken production of complex sentences in 8- and 12-year-old children and adults. Journal of Experimental Psychology. General, 144(2), 447-468.

Newport, E. L., \& Aslin, R. N. (2004). Learning at a distance I. Statistical learning of non-adjacent dependencies. Cognitive Psychology, 48(2), 127-162.

Pallier, C. (2002). Computing discriminability and bias with the $R$ software. http://www.pallier.org/pdfs/aprime.pdf

Pinker, S. (1984). Language learnability and language development. Harvard University Press.

Plaut, D. C., \& Vande Velde, A. K. (2017). Statistical learning of parts and wholes: A neural network approach. Journal of Experimental Psychology. General, 146(3), 318-336.

Rastle, K., Harrington, J., \& Coltheart, M. (2002). 358,534 nonwords: The ARC nonword database. The Quarterly Journal of Experimental Psychology Section A, 55, 1339-1362.

R Core Team. (2013). R: A language and environment for statistical computing. Vienna, Austria. http://www.R-project.org/ 
Redington, M., Chater, N., \& Finch, S. (1998). Distributional information: a powerful cue for acquiring syntactic categories. Cognitive Science, 22, 425-469.

Reeder, P. A., Newport, E. L., \& Aslin, R. N. (2013). From shared contexts to syntactic categories: the role of distributional information in learning linguistic form-classes. Cognitive Psychology, 66(1), 30-54.

Reeder, P. A., Newport, E. L., \& Aslin, R. N. (2017). Distributional learning of subcategories in an artificial grammar: Category generalization and subcategory restrictions. Journal of Memory and Language, 97, 17-29.

Romberg, A. R., \& Saffran, J. R. (2013). All together now: concurrent learning of multiple structures in an artificial language. Cognitive Science, 37(7), 1290-1320.

Saffran, J. R., Newport, E. L., \& Aslin, R. N. (1996). Word Segmentation: The Role of Distributional Cues. Journal of Memory and Language, 35(4), 606-621.

Saffran, J. R., Newport, E. L., Aslin, R. N., Tunick, R. A., \& Barrueco, S. (1997). Incidental Language Learning: Listening (and Learning) Out of the Corner of Your Ear. Psychological Science, 8(2), 101-105.

Sawi, O. M., \& Rueckl, J. (2019). Reading and the Neurocognitive Bases of Statistical Learning. Scientific Studies of Reading: The Official Journal of the Society for the Scientific Study of Reading, 23(1), 8-23.

Seidenberg, M. S., \& MacDonald, M. C. (2018). The Impact of Language Experience on Language and Reading: A Statistical Learning Approach. Topics in Language Disorders, 38(1), 66.

Shi, R., Morgan, J., \& Allopenna, P. (1998). Phonological and acoustic basis for earliest grammatical category assignment: a cross-linguistic perspective. Journal of Child Language, $25,169-201$.

Siegelman, N., Rueckl, J. G., Steacy, L. M., Frost, S. J., van den Bunt, M., Zevin, J. D., Seidenberg, M. S., Pugh, K. R., Compton, D. L., \& Morris, R. D. (2020). Individual differences in learning the regularities between orthography, phonology and semantics predict early reading skills. In Journal of Memory and Language (Vol. 114, p. 104145). https://doi.org/10.1016/j.jml.2020.104145

Smalle, E. H. M., Page, M. P. A., Duyck, W., Edwards, M., \& Szmalec, A. (2018). Children retain implicitly learned phonological sequences better than adults: a longitudinal study. Developmental Science, 21(5), e12634.

Smith, L. B., Suanda, S. H., \& Yu, C. (2014). The unrealized promise of infant statistical wordreferent learning. Trends in Cognitive Sciences, 18(5), 251-258.

Smith, L. B., \& Yu, C. (2008). Infants rapidly learn word-referent mappings via cross-situational statistics. Cognition, 106(3), 1558-1568.

Spencer, M., Kaschak, M. P., Jones, J. L., \& Lonigan, C. J. (2015). Statistical Learning is Related to 
Early Literacy-Related Skills. Reading and Writing, 28(4), 467-490.

Tamminen, J., Davis, M. H., Merkx, M., \& Rastle, K. (2012). The role of memory consolidation in generalisation of new linguistic information. Cognition, 125(1), 107-112.

Turk-Browne, N. B., Isola, P. J., Scholl, B. J., \& Treat, T. A. (2008). Multidimensional visual statistical learning. Journal of Experimental Psychology. Learning, Memory, and Cognition, 34(2), 399-407.

Ulicheva, A., Harvey, H., Aronoff, M., \& Rastle, K. (2020). Skilled readers' sensitivity to meaningful regularities in English writing. Cognition, 195, 103810.

van Kesteren, M. T. R., Ruiter, D. J., Fernández, G., \& Henson, R. N. (2012). How schema and novelty augment memory formation. Trends in Neurosciences, 35(4), 211-219.

Vlach, H. A., Ankowski, A. A., \& Sandhofer, C. M. (2012). At the same time or apart in time? The role of presentation timing and retrieval dynamics in generalization. Journal of Experimental Psychology. Learning, Memory, and Cognition, 38(1), 246-254.

Vouloumanos, A. (2008). Fine-grained sensitivity to statistical information in adult word learning. Cognition, 107(2), 729-742.

Wechsler, D. (2011). WASI-II: Wechsler abbreviated scale of intelligence. PsychCorp.

Weighall, A. R., Henderson, L., Barr, D. J., Cairney, S. A., \& Gaskell, G. M. (2017). Eye-tracking the time-course of novel word learning and lexical competition in adults and children. Brain and Language, 167, 13-27.

Wells, J. B., Christiansen, M. H., Race, D. S., Acheson, D. J., \& MacDonald, M. C. (2009). Experience and sentence processing: statistical learning and relative clause comprehension. Cognitive Psychology, 58(2), 250-271.

Werchan, D. M., \& Gómez, R. L. (2014). Wakefulness (not sleep) promotes generalization of word learning in 2.5-year-old children. Child Development, 85(2), 429-436.

Wonnacott, E. (2011). Balancing generalization and lexical conservatism: An artificial language study with child learners. Journal of Memory and Language, 65(1), 1-14.

Wonnacott, E., Boyd, J. K., Thomson, J., \& Goldberg, A. E. (2012). Input effects on the acquisition of a novel phrasal construction in 5year olds. Journal of Memory and Language, 66(3), 458478.

Wonnacott, E., Brown, H., \& Nation, K. (2017). Skewing the evidence: The effect of input structure on child and adult learning of lexically based patterns in an artificial language. Journal of Memory and Language, 95, 36-48.

Yu, C., \& Smith, L. B. (2007). Rapid word learning under uncertainty via cross-situational statistics. Psychological Science, 18(5), 414-420.

Yu, C., \& Smith, L. B. (2011). What you learn is what you see: using eye movements to study infant cross-situational word learning. Developmental Science, 14(2), 165-180. 
Zamuner, T. S., Morin-Lessard, E., Strahm, S., \& Page, M. P. A. (2016). Spoken word recognition of novel words, either produced or only heard during learning. Journal of Memory and Language, 89, 55-67.

Zubin, D. A., \& Köpcke, K. M. (1981). Gender: A less than arbitrary grammatical category. In R. A. Hendrick, C. A. Masek, \& M. F. Miller (Eds.), Papers from the seventeenth regional meeting, Chicago Linguistic Society (pp. 439-449). Chicago Linguistic Society.

Zubin, D. A., \& Köpcke, K. M. (1986). Gender and folk taxonomy: The indexical relation between grammatical and lexical categorization. In C. G. Craig (Ed.), Noun classes and categorization: Proceedings of a symposium on categorization and noun classification, Eugene, Oregon, October 1983 (pp. 139-180). Benjamins. 